Review Article

\title{
Mechanisms of Herbal Nephroprotection in diabetes mellitus
}

\author{
Dorin Dragoș $\mathbb{D},{ }^{1,2}$ Maria Mirabela Manea $\mathbb{D}^{1,3}$ Delia Timofte $\mathbb{D}^{1,4}$ and Dorin Ionescu $\mathbb{D}^{1,2}$ \\ ${ }^{1}$ Faculty of General Medicine, "Carol Davila” University of Medicine and Pharmacy, str. Dionisie Lupu nr. 37, sect 1, \\ Bucharest 020021, Romania \\ ${ }^{2}$ Nephrology Clinic of University Emergency Hospital, Splaiul Independentei nr. 169, sect. 5, Bucharest 050098, Romania \\ ${ }^{3}$ National Institute of Neurology and Cerebrovascular Diseases, Şos. Berceni, Nr. 10-12, Sector 4, Bucharest 041914, Romania \\ ${ }^{4}$ Dialysis Department of University Emergency Hospital, Splaiul Independentei $\mathrm{nr} .169$, sect. 5, Bucharest 050098, Romania
}

Correspondence should be addressed to Maria Mirabela Manea; manea.maria.mirabela@gmail.com

Received 28 January 2020; Revised 24 May 2020; Accepted 6 June 2020; Published 6 July 2020

Academic Editor: Eric Hajduch

Copyright ( 2020 Dorin Dragoș et al. This is an open access article distributed under the Creative Commons Attribution License, which permits unrestricted use, distribution, and reproduction in any medium, provided the original work is properly cited.

\begin{abstract}
Diabetic nephropathy (DN) is a leading cause of kidney morbidity. Despite the multilayered complexity of the mechanisms involved in the pathogenesis of $\mathrm{DN}$, the conventional treatment is limited to just a few drug classes fraught with the risk of adverse events, including the progression of renal dysfunction. Phytoceuticals offer a promising alternative as they act on the many-sidedness of DN pathophysiology, multitargeting its intricacies. This paper offers a review of the mechanisms underlying the protective action of these phytoagents, including boosting the antioxidant capabilities, suppression of inflammation, averting the proliferative and sclerosing/fibrosing events. The pathogenesis of $\mathrm{DN}$ is viewed as a continuum going from the original offense, high glucose, through the noxious products it generates (advanced glycation end-products, products of oxidative and nitrosative stress) and the signaling chains consequently brought into action, to the harmful mediators of inflammation, sclerosis, and proliferation that eventually lead to DN, despite the countervailing attempts of the protective mechanisms. Special attention was given to the various pathways involved, pointing out the ability of the phytoagents to hinder the deleterious ones (especially those leading to, driven by, or associated with TGF- $\beta$ activation, SREBP, Smad, MAPK, PKC, NF- $\kappa$ B, NLRP3 inflammasome, and caspase), to promote the protective ones (PPAR- $\alpha$, PPAR- $\gamma$, EP4/Gs/AC/cAMP, Nrf2, AMPK, and SIRT1), and to favorably modulate those with potentially dual effect (PI3K/Akt). Many phytomedicines have emerged as potentially useful out of in vitro and in vivo studies, but the scarcity of human trials seriously undermines their usage in the current clinical practice - an issue that stringently needs to be addressed.
\end{abstract}

\section{Introduction}

In most countries, diabetic nephropathy (DN) (also known as diabetic kidney disease) is the main cause of chronic kidney disease (CKD) [1]. DN results from the interplay of several distinct but highly interconnected high glucose- (HG-) induced pathways set into motion by aggressive factors, such as oxidative stress [2] and advanced glycation end-products (AGEs), which trigger signaling chains that generate mediators able to instigate reactive processes, including inflammation, cellular proliferation, and interstitial matrix expansion [3]. Oxidative stress and inflammation enhance each other, resulting in a vicious circle leading to glomerular sclerosis and interstitial fibrosis [4]. Figure 1 illustrates some of the most important mechanisms of $\mathrm{DN}$, although it is by no means exhaustive.

Among the inflammatory mediators involved in DN are nuclear factor kappa-B (NF- $\kappa \mathrm{B})$, monocyte chemotactic protein- (MCP-) 1, and intercellular adhesion molecule(ICAM-) 1. Attracted and activated by MCP-1 and helped by ICAM-1 (promoted by NF- $\kappa \mathrm{B}$ ), circulating monocytes invade the kidney [5]. Together with resident mesangial cells proliferation, this leads to the mesangial hypercellularity characteristic for the diabetic glomerulus [6]. Glomerulosclerosis is the hallmark of DN [7] and consists in proteins of extracellular matrix (ECM) (mostly collagen types I, III, and IV and fibronectin [8]) gradually and inexorably encumbering the mesangium, either by lumping together in nodular 


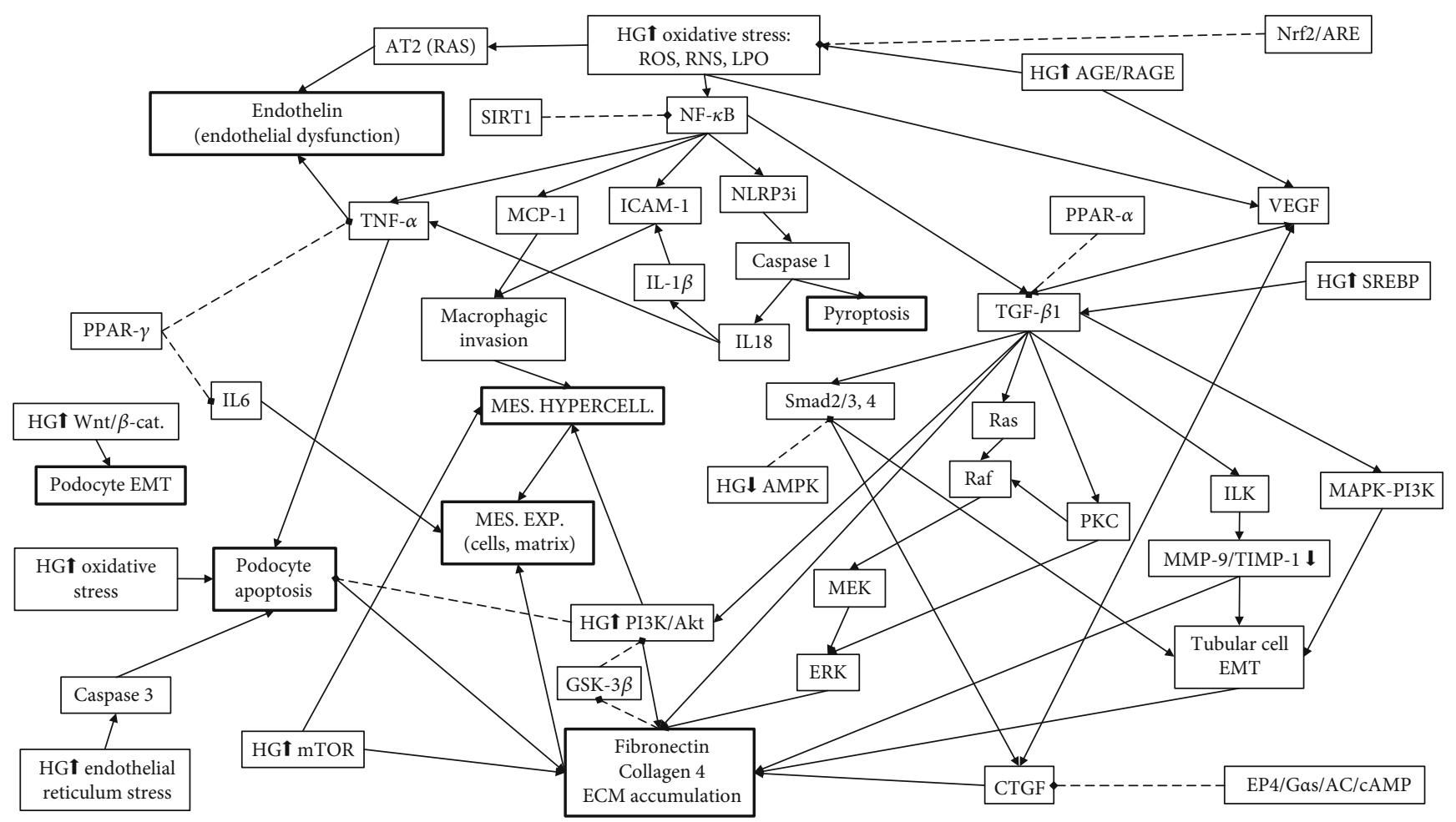

FIGURE 1: Pathways and mediators of DN relevant for the action mechanisms of the phytoagents active in DN. The schematic is meant to highlight the complexity of the factors and interconnections involved in DN pathogenesis, although it leaves out many of them lest it might become unintelligible. HG $\uparrow$ : activated/increased/induced by high glucose (HG); HG $\downarrow$ : inactivated/decreased by $H G$, mes.: mesangial; exp.: expansion; hypercell.: hypercellularity; continuous arrowhead-ended lines indicate a stimulating effect; dotted diamondended lines indicate an inhibitory effect.

lesions or by diffusely invading and expanding the interstitial space separating the glomerular loops [9]. The ECM proteins accumulation is the result of excessive production (mostly by mesangial cells) coupled with insufficient proteolysis by mesangial matrix metalloproteinases (MMPs) [10]. However, the pathological deposition of ECM proteins is not limited to the glomerular interstitium but also involves the tubular interstitium and the glomerular basement membrane, explaining its increased thickness [8]. The cellular component of both glomerulus and tubulointerstitium is altered by epithelial-mesenchymal transdifferentiation (EMT) [4]. Growth-promoting pathways involved in DN are those driven by p38 mitogen-activated protein kinase (p38MAPK) [11], mammalian target of rapamycin (mTOR), and phosphatidylinositol 3-kinase (PI3K)/Akt/glycogen synthase kinase- (GSK-) $3 \beta$, the last two sometimes acting in concert [12]. It is not clear whether PI3K/Akt activation should be considered protective or deleterious [13]. Endothelial dysfunction [14] and endoplasmic reticulum (ER) stress are other important links in the pathophysiological chain leading to DN $[15,16]$.

Among the pharmaceuticals used for slowing the progression of DN are lipid-lowering agents and renin-angiotensinaldosterone system blockers, especially angiotensin-converting enzyme inhibitors and angiotensin receptor blockers [17], but also mineralocorticoid receptor antagonists [18]. However, the use of these drugs is fraught with the risk of adverse events, including liver and muscle injury, progression of renal dysfunction, and hyperkalemia. Consequently, therapeutic alternatives devoid of such adverse effects are needed. Worldwide herbs have been used for the treatment of diabetes [19], including in the two most practiced systems of traditional medicine: ayurvedic [20] and traditional Chinese medicine [21]. Due to the complexity of their composition, medicinal herbs have, over the modern antidiabetic drugs, the advantage of influencing multiple pathogenic mechanisms $[22,23]$ and being devoid of significant adverse effects [24]. Herbal treatment can complement and even increase the efficiency of the conventional one, the synergistic effect of the combined treatment allowing for dose reduction and delaying the need for insulin therapy [23]. The aim of this article is to review the mechanisms underlying the protective action of these phytomedicines.

\section{Material and Method}

A PubMed (https://www.ncbi.nlm.nih.gov/pubmed) search for ("diabetic nephropathy"[Title/Abstract] OR "diabetic kidney disease"[Title/Abstract]) AND (plant[Title/Abstract] OR herbal[Title/Abstract]) and a ScienceDirect (https:// www.sciencedirect.com/) search for ("diabetic nephropathy" OR "diabetic kidney disease") AND (plant OR herbal) provided the articles employed in this review. The authors endeavored to include (almost) all the relevant papers, preferring those attempting to define the molecular/cellular background of the renoprotective action and those investigating 
clearly defined active compounds or herbal products (articles on herbal formulas with unclear components were left out). The results of this search are summarized in Table 1.

We considered that a study proved that a herbal product is actually nephroprotective if it demonstrated lower levels of glomerular injury markers (in most cases, albuminuria and/or proteinuria) or improved kidney histology or function (stated as such or reflected by lower serum levels of urea and/or creatinine) in the subjects who took the herbal product. We considered the herbal products for which neither of these effects was demonstrated as only potentially nephroprotective. Practically all the in vivo studies (there was only one exception), done on either human or animal subjects (including those that also had an in vitro component), proved the ability of the investigated phytoceuticals to protect the kidney, while none of the exclusively in vitro studies did so. Consequently, for each mechanism or pathway, the data were separated into in vitro, in vivo, and clinical, pointing out that the in vitro studies suggest potentially protective mechanisms, while the in vivo ones demonstrate actually protective mechanisms.

In most of the in vivo studies, nephroprotection consisted in the ability of the phytoceuticals to lessen the degree of glomerular and/or tubulointerstitial injury at the completion of the study, which may be equated with the ability to delay the progression of DN. No study could demonstrate the complete prevention of $\mathrm{DN}$; therefore, none of the plant products was able to prevent the onset of $\mathrm{DN}$.

In order to assess the strength of the evidence in the area of the clinical trials regarding the efficiency of herbals in DN, a secondary search was performed: on PubMed for ("diabetic nephropathy"[Title/Abstract] OR "diabetic kidney disease"[Title/Abstract]) AND (plant[Title/Abstract] OR herbal[Title/Abstract]) AND (trial[Title/Abstract]) and on ScienceDirect for ("diabetic nephropathy" OR "diabetic kidney disease") AND (plant OR herbal) AND (trial). The Jadad scale was employed for evaluating the quality of the human trials [25]. The trials with Jadad score of 1, 2, 3, 4, and 5 (designated as J1, J2, J3, J4, and J5, respectively) were considered of low, low-to-moderate, moderate, moderate-to-high, and high quality, respectively.

\section{Herbal Nephroprotection}

3.1. Herbal Products Decreasing Serum Glucose Level and Peripheral Resistance. As HG is the point of depart in the pathophysiological chain leading to DN, optimal glycemic control prevents CKD or at least delays its onset and slows its pace [26]. Insulin resistance may be involved in the genesis of $\mathrm{DN}[27]$.

3.1.1. In Vivo Studies-Actually Nephroprotective Mechanisms. Many of the investigated herbal products are able to decrease glucose level (Allium sativum [28], Artemisia sieberi [29], Bacopa monnieri [30], Hypericum perforatum [31], Punica granatum [32], and Terminalia chebula [33] to name only a few-see Table 2 for a complete list) and some of them are also able to decrease insulin resistance (Cladophora glomerata [34], Panax notoginseng [35], Huangqi decoction [36] etc.-see Table 2) which corroborates or explains their glucose-lowering effect. This is evident especially on the animal models conceived to mimic as good as possible the actual human disease, such as high-fat diet/low-dose streptozotocin type 2 diabetic Wistar albino rats in which some of the most important features of human type 2 diabetes are recognizable: hyperglycemia, insulin resistance, heightened oxidative stress, and structural and functional kidney deterioration. In this animal model, obesity-related insulin resistance, and not lack of insulin, is the cause of altered glucose metabolism. Insulin resistance (that may be attenuated by ellagic acid) is the result of the inflammatory response triggered by adipose tissue infiltration by immune cells spurred by proinflammatory cytokines such as interleukin- (IL-) $1 \beta$, IL-6, and tumor necrosis factor- (TNF-) $\alpha$ produced by NF- $\kappa \mathrm{B}$-activated adipocytes [37].

\subsection{Herbal Products Decreasing Oxidative Stress and AGEs} Production. Oxidative stress is the consequence of antioxidant protective mechanisms being overwhelmed by the reactive oxygen species (ROS) generation. It leads to reactive nitrogen species (such as peroxynitrite) production, lipid peroxidation, inflammatory pathways activation [via NF$\kappa \mathrm{B}$, protein kinase $\mathrm{C}(\mathrm{PKC})$, etc.], apoptosis, and mesangium (both cells and matrix) expansion [4,38]. Acting by means of RAGE (receptor for AGEs), AGEs induce inflammation, oxidative stress, apoptosis, and exuberant ECM protein synthesis [39], one of the mediators being ROS production [8].

\subsubsection{In Vitro Studies-Potentially Nephroprotective} Mechanisms. Paeoniflorin and oxypaeoniflora (from Paeonia suffruticosa), improved the protection against AGE-induced inflammatory and oxidative damage by boosting glutathione peroxidase and catalase activities, hampering both the cellular (macrophage migration), and humoral (transcription factors, cytokines) components of inflammation [40]. Curcumin and demethoxycurcumin (from Curcuma longa) prevented AGE-induced apoptosis of mesangial cells [41].

Oxidative stress also activates the inflammatory response and undermines cell viability. Consequently, moringa isothiocyanate (from Moringa oleifera), able to activate nuclear factor erythroid-derived 2 (Nrf2), the chief regulator of the antioxidant response, also suppresses transforming growth factor- $\beta 1$ (TGF- $\beta 1$ ) signaling and the production of proinflammatory cytokines by macrophages [42]. Silybin (from Silybum marianum) preserves the viability of HG-injured podocytes by decreasing superoxide production, reduced nicotinamide-adenine dinucleotide phosphate (NADPH) oxidase activity, and NAPDH oxidase 4 (NOX4) expression [43].

The PI3K/Akt pathway increases insulin sensitivity, averts apoptosis, and is important for recovery after kidney injury, but it may also deleteriously promote cell proliferation and fibrogenesis in DN. A similar action may have $m T O R$ pathway, and these two pathways may act in concert. Inactivating phosphorylation of GSK- $3 \beta$ may be one of the mechanisms mediating the proliferative and fibrogenetic effects of PI3K/Akt activation [12]. Berberine (from Berberis vulgaris) induces PI3K/Akt signaling pathway, which results in the activation of Nrf2 (and its target genes, including heme 


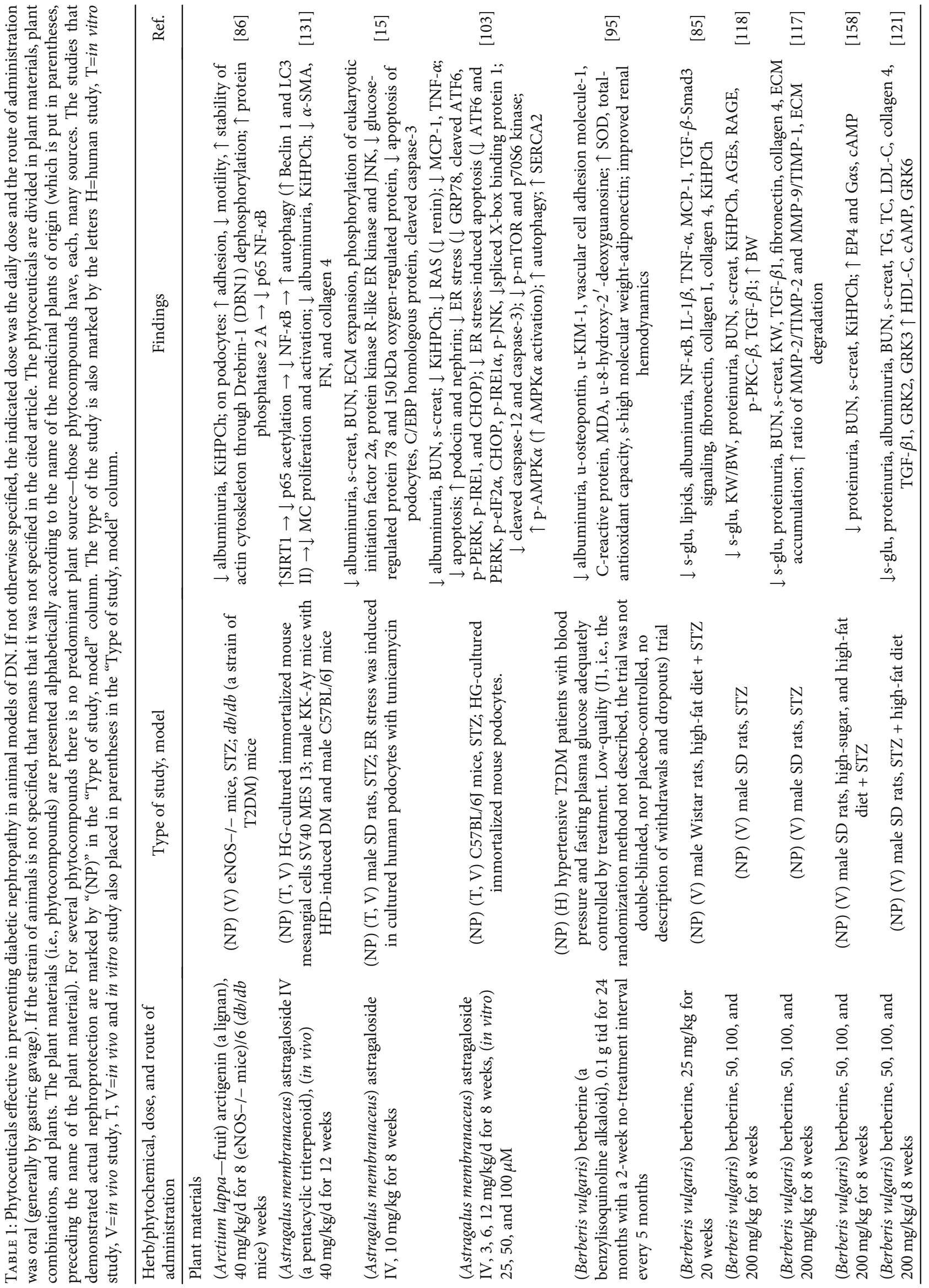




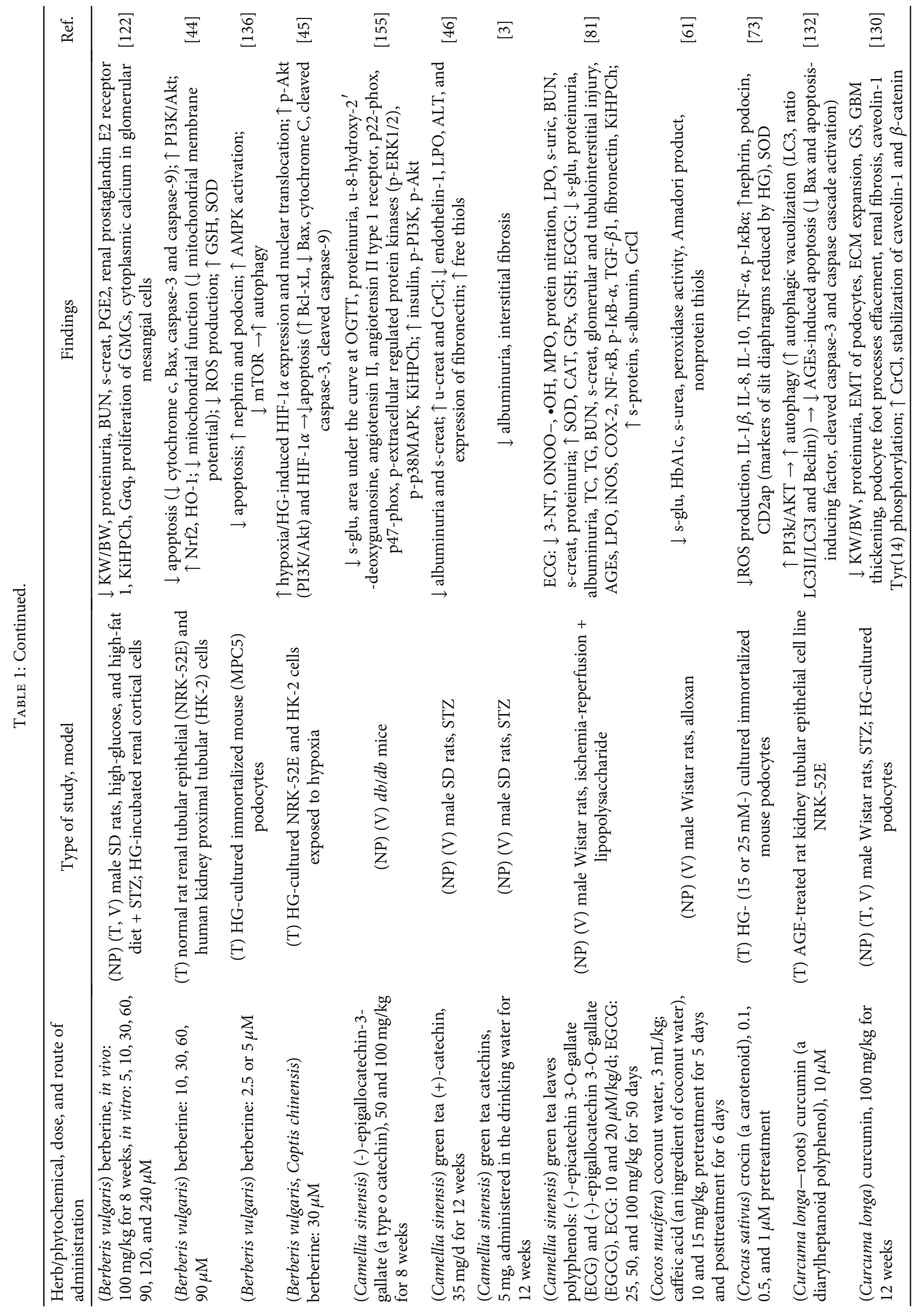




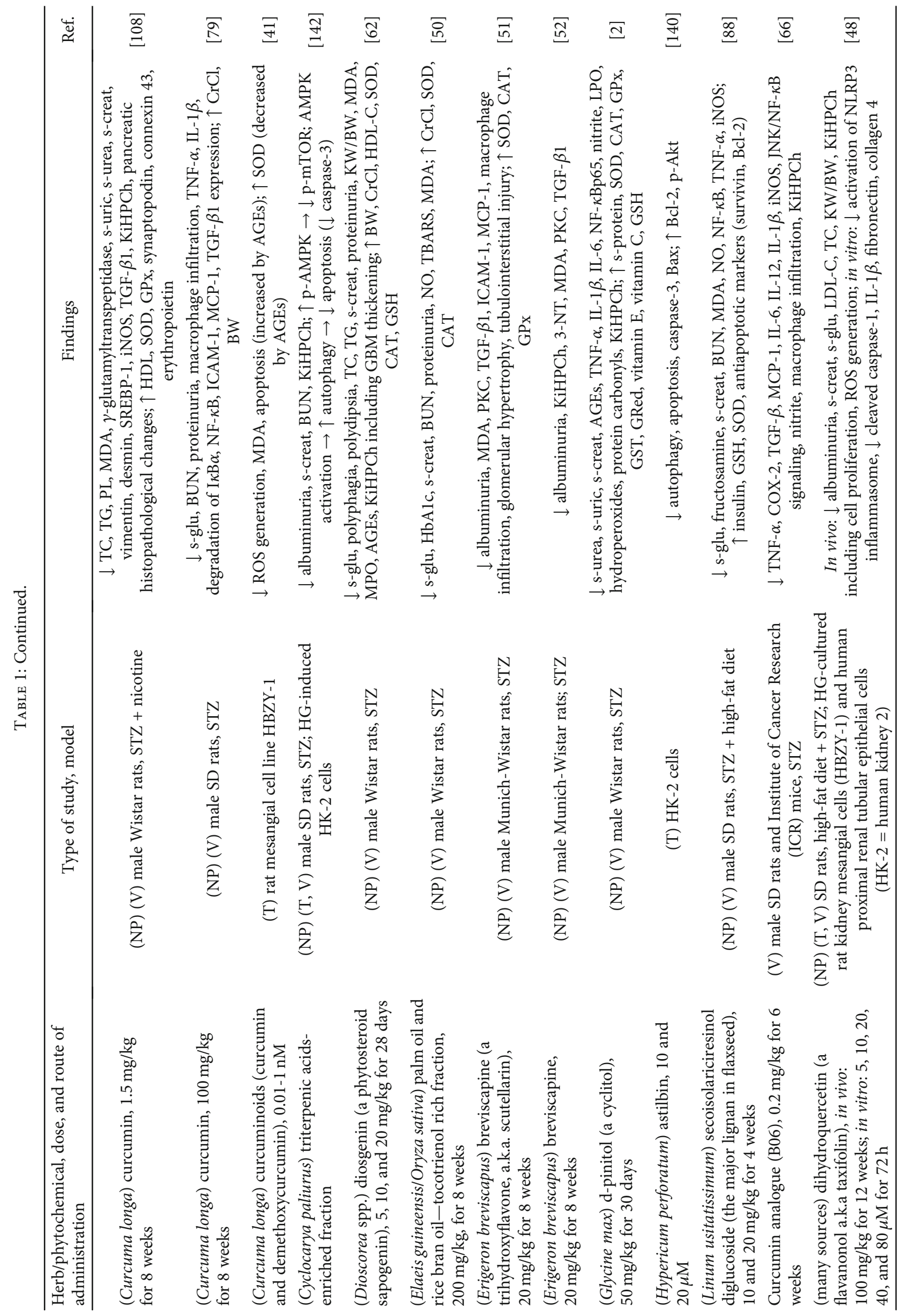




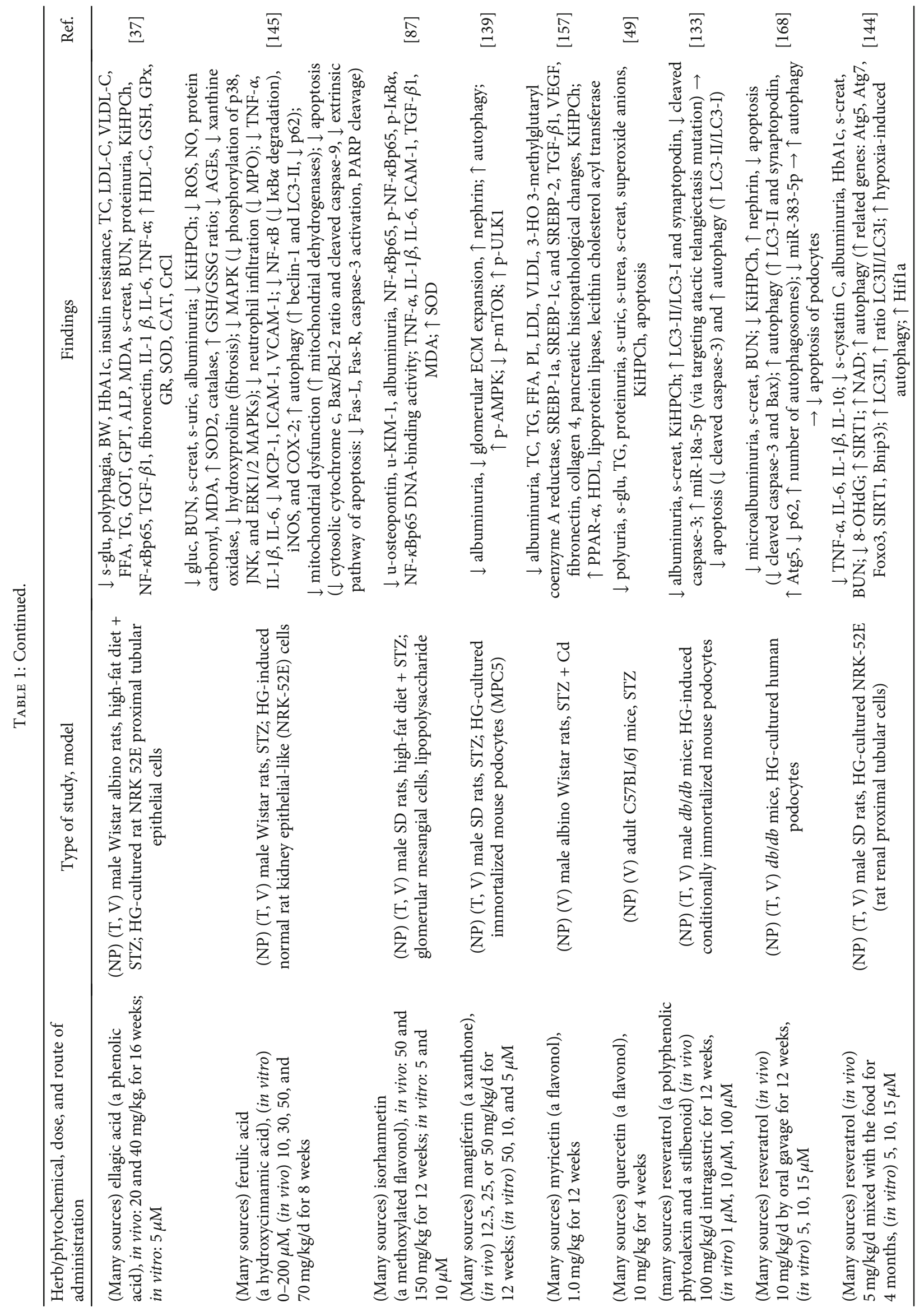




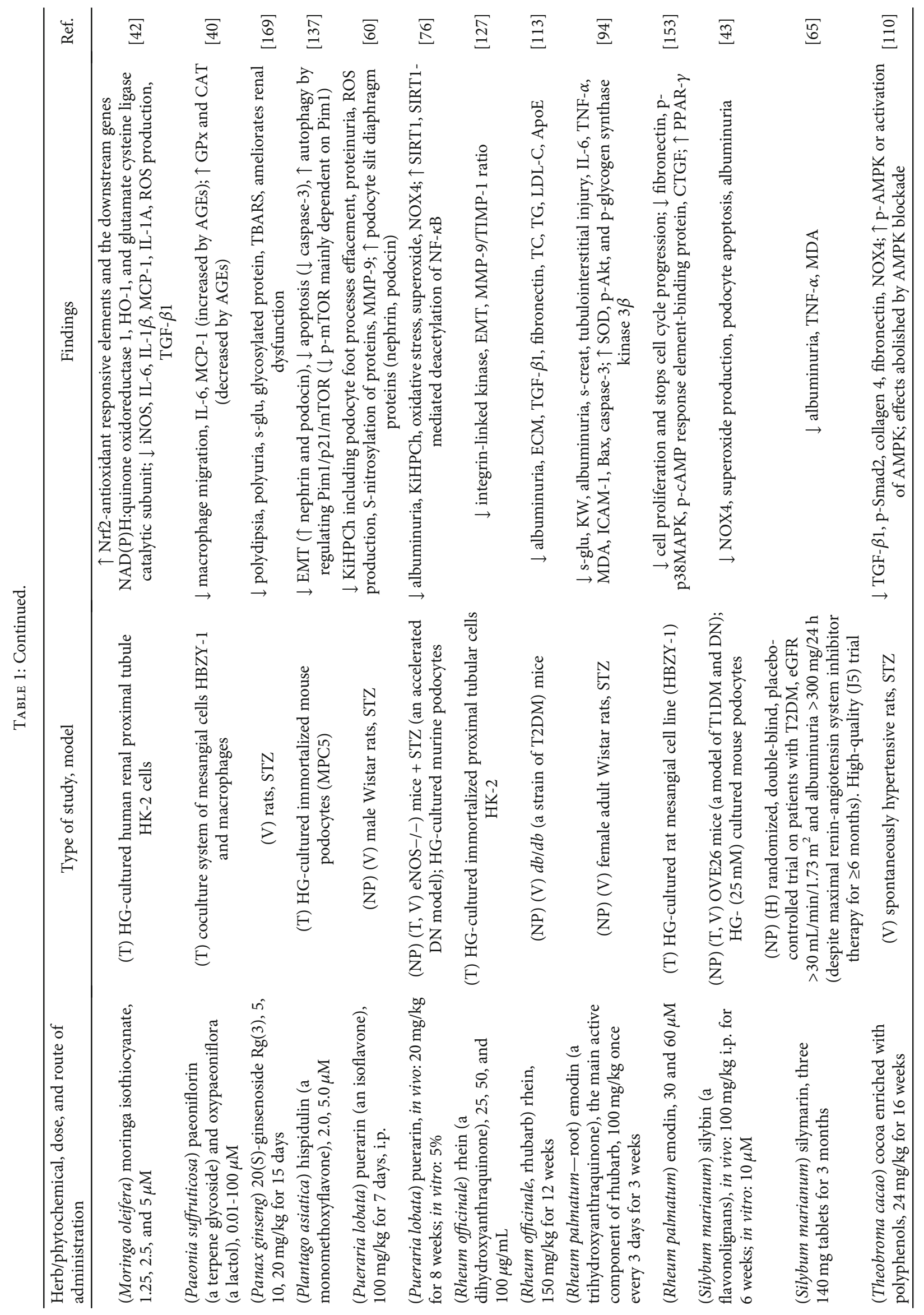




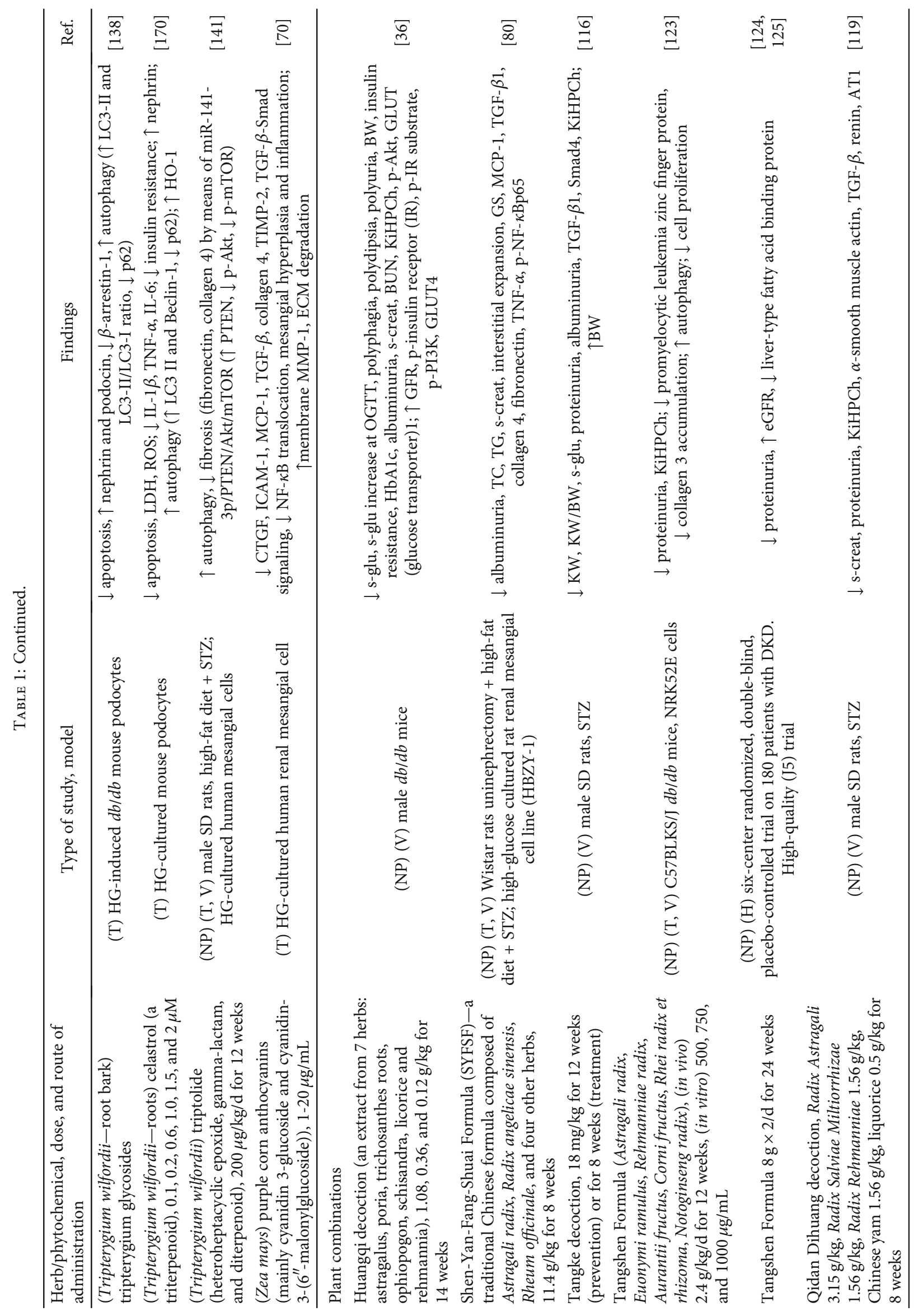




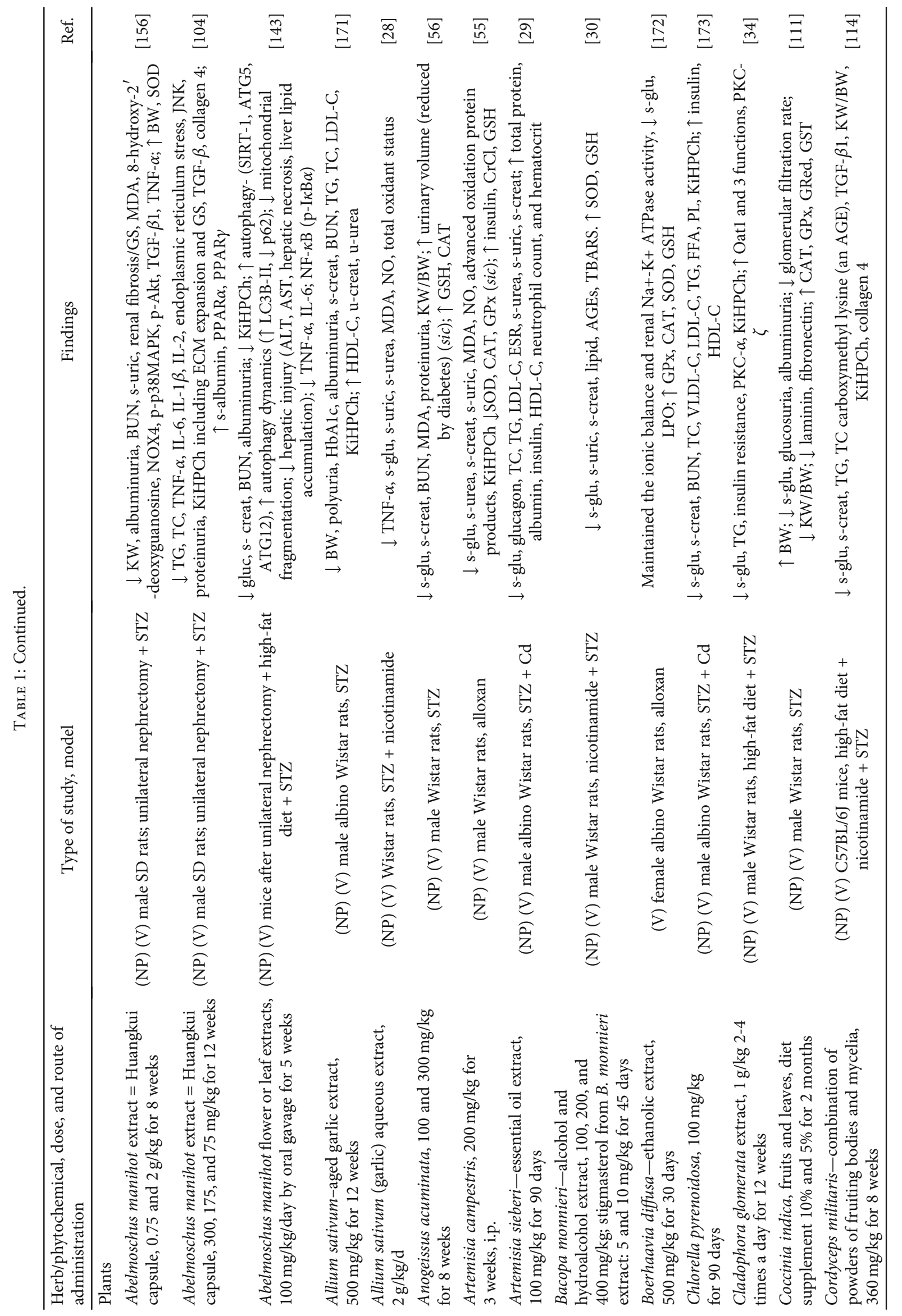




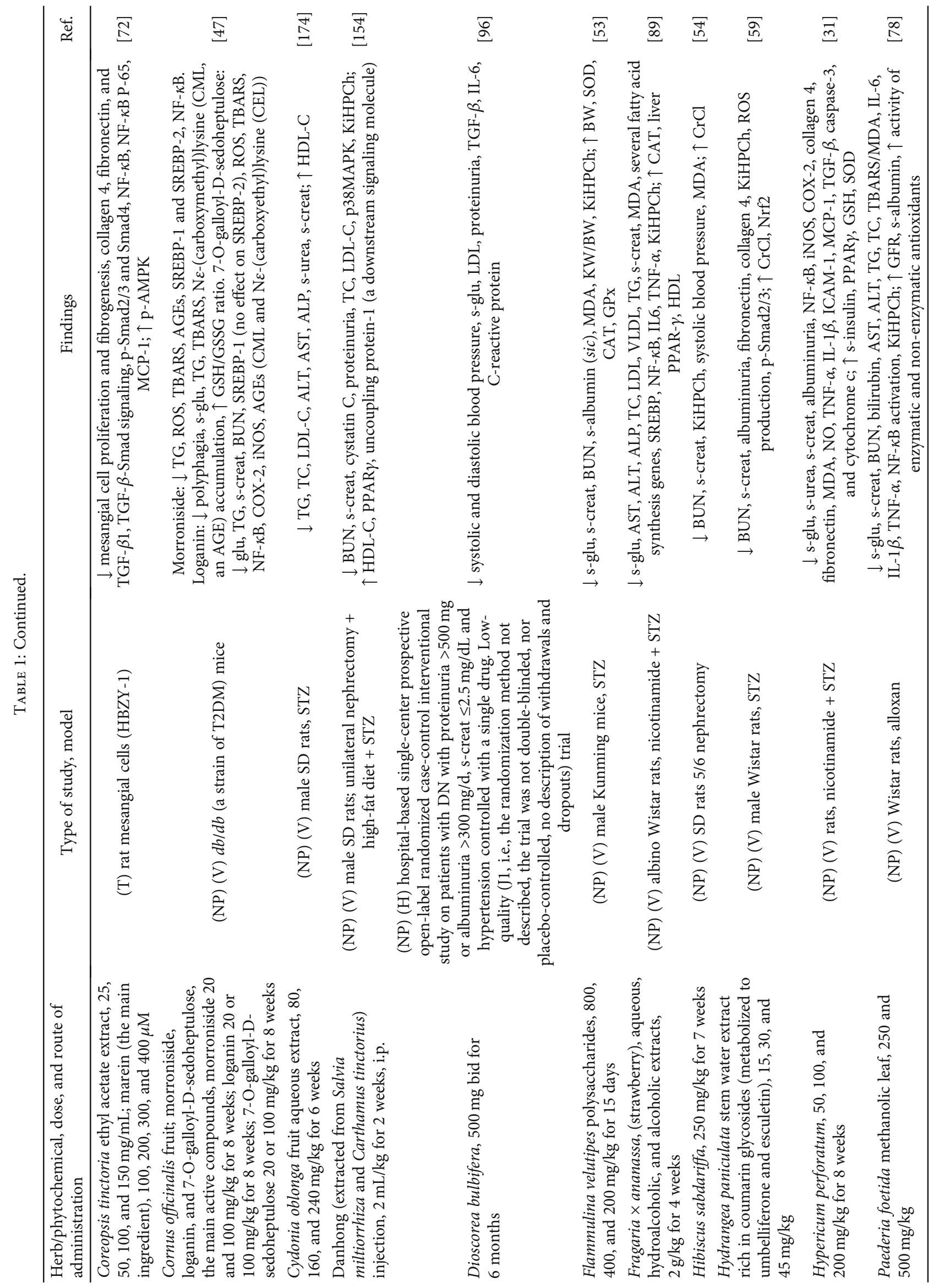




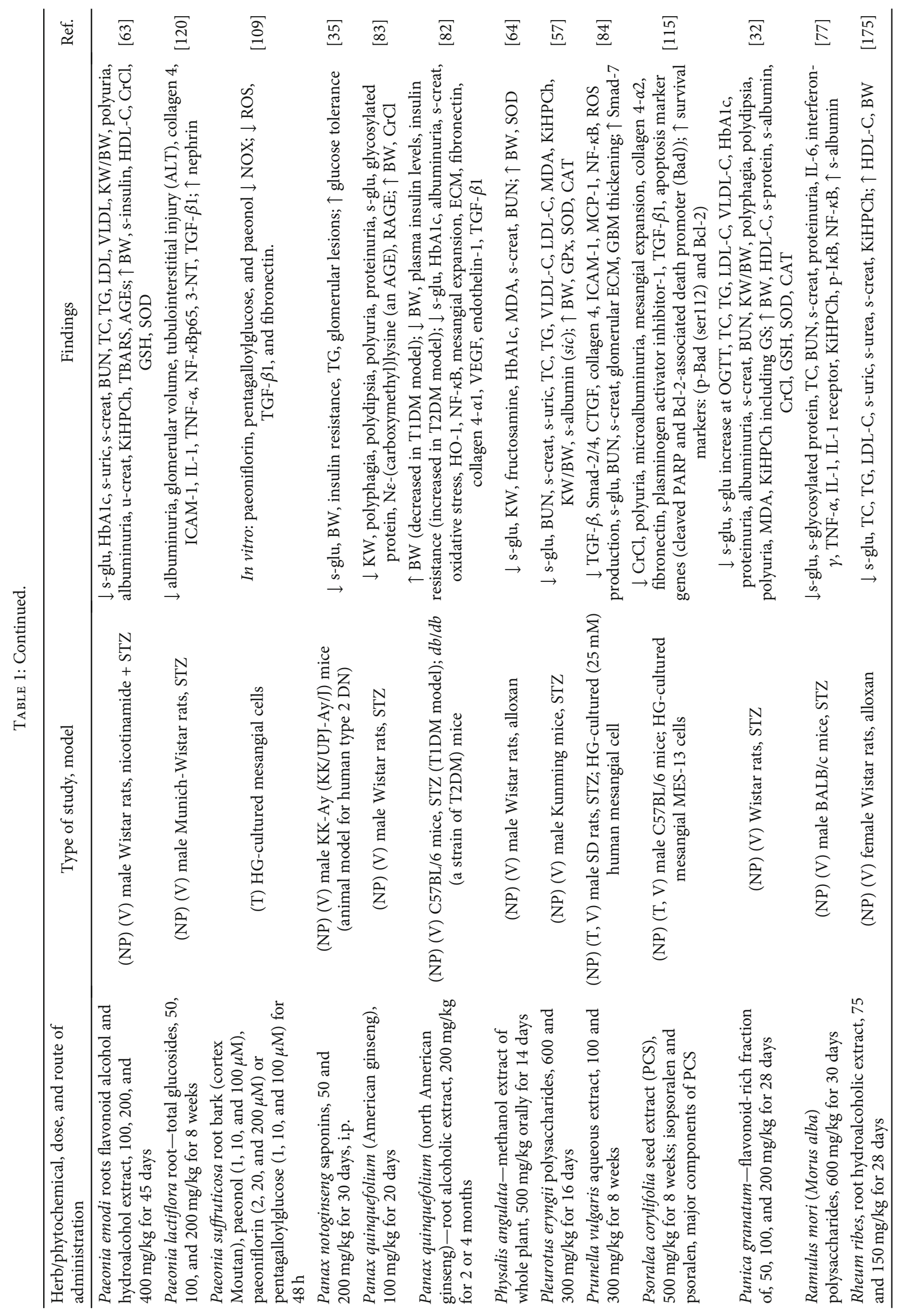




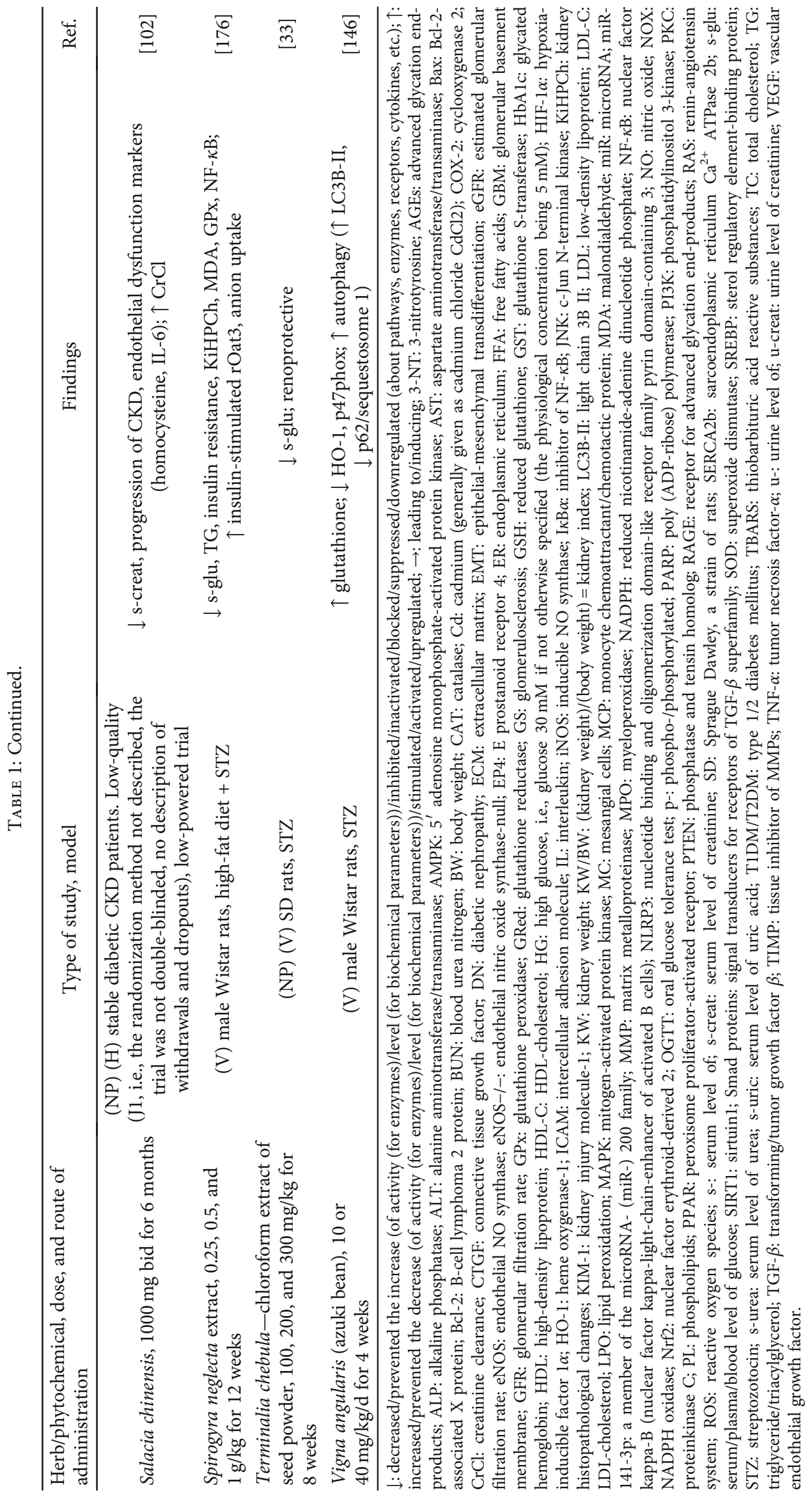


TABLE 2: Phytoagents active in diabetic nephropathy classified according to their mechanism of action. The marker (H) was used to signal human studies.

\begin{tabular}{|c|c|}
\hline \multicolumn{2}{|l|}{ Aggressive factors } \\
\hline & Glucose metabolism \\
\hline Serum glucose level & $\begin{array}{l}\text { (-)-Epicatechin 3-O-gallate and (-)-epigallocatechin 3-O-gallate [81], } \\
\text { (-)-epigallocatechin-3-gallate [155], 20(S)-ginsenoside Rg(3) [169], Allium } \\
\text { sativum [28], Anogeissus acuminata [56], Artemisia campestris [55], Artemisia } \\
\text { sieberi [29], Bacopa monnieri [30], berberine [85] [117] [118] [121], Boerhaavia } \\
\text { diffusa [172], Chlorella pyrenoidosa [173], Cladophora glomerata [34], Coccinia } \\
\text { indica [111], coconut water [61], Cordyceps militaris [114], Cornus officinalis } \\
\text { [47], curcumin [79], dihydroquercetin [48], Dioscorea bulbifera [96], diosgenin } \\
\text { [62], ellagic acid [37], emodin\$ [94], Flammulina velutipes [53], Fragaria } \times \\
\text { ananassa [89], Huangqi decoction [36], Hypericum perforatum [31], Paederia } \\
\text { foetida [78], Paeonia emodi [63], palm oil and rice bran oil [50], Panax } \\
\text { notoginseng [35], Panax quinquefolium [82], Panax quinquefolium [83], Physalis } \\
\text { angulata [64], Pleurotus eryngii polysaccharides [57], Prunella vulgaris [84], } \\
\text { Punica granatum [32], quercetin [49], Ramulus mori [77], Rheum ribes [175], } \\
\text { secoisolariciresinol diglucoside [88], Spirogyra neglecta [176], Tangke decoction } \\
\text { [116], Terminalia chebula [33] }\end{array}$ \\
\hline Insulin resistance & $\begin{array}{l}\text { Cladophora glomerata [34], ellagic acid [37], Huangqi decoction [36], Panax } \\
\text { notoginseng [35], Panax quinquefolium [82], Spirogyra neglecta [176] }\end{array}$ \\
\hline
\end{tabular}

Oxidative/nitrosative stress

(-)-Epigallocatechin-3-gallate [155], Abelmoschus manihot [156], Allium sativum [28], Artemisia campestris [55], berberine [95] (human), berberine [44], celastrol [170], cocoa enriched with polyphenols [110], Cornus officinalis [47], crocin [73],

Oxidative stress curcumin [41], dihydroquercetin [48], d-pinitol [2], ferulic acid [145], Hydrangea paniculata [59], moringa isothiocyanate [42], Paeonia suffruticosa [109], Panax quinquefolium [82], Prunella vulgaris [84], puerarin [76], puerarin [60], quercetin [49], silybin [43], Vigna angularis [146]

(-)-Epicatechin 3-O-gallate and (-)-epigallocatechin 3-O-gallate [81], (+)-catechin [46], Abelmoschus manihot [156], Allium sativum [28], Anogeissus acuminata [56], Artemisia campestris [55], Bacopa monnieri [30], berberine [95] (human), Boerhaavia diffusa [172], breviscapine [51], breviscapine [52], Cornus officinalis [47], curcumin [108], curcumin [41], diosgenin [62], d-pinitol [2],

Lipid peroxidation (LPO) ellagic acid [37], emodin [94], ferulic acid [145], Flammulina velutipes [53], Fragaria $\times$ ananassa [89], Hibiscus sabdariffa [54], Hypericum perforatum [31], isorhamnetin [87], Paederia foetida [78], Paeonia emodi [63], Paeonia suffruticosa [40], Panax ginseng [169], Physalis angulata [64], Pleurotus eryngii [57], Punica granatum [32], secoisolariciresinol diglucoside [88], silymarin [65] (human), Spirogyra neglecta [176]

(-)-Epicatechin 3-O-gallate and (-)-epigallocatechin 3-O-gallate [81], Abelmoschus manihot [156], Allium sativum [28], Artemisia campestris [55], breviscapine [52], cocoa enriched with polyphenols [110], Cornus officinalis [47],

Nitrosative stress curcumin [108], curcumin analogue (B06) [66], d-pinitol [2], Hypericum perforatum [31], moringa isothiocyanate [42], Paeonia lactiflora [120], Paeonia suffruticosa [40], puerarin [76], puerarin [60], secoisolariciresinol diglucoside [88], silybin [43], ferulic acid [145]

Antioxidant protection

Antioxidant capacity

Berberine [95] (human), Paederia foetida [78]

(-)-Epicatechin 3-O-gallate and (-)-epigallocatechin 3-O-gallate [81], Anogeissus acuminata [56], Artemisia campestris [55], Boerhaavia diffusa [172], breviscapine

Catalase (CAT) [51], Coccinia indica [111], diosgenin [62], d-pinitol [2], ellagic acid [37], Flammulina velutipes [53], Fragaria $\times$ ananassa [89], Paeonia suffruticosa [40], Pleurotus eryngii [57], Punica granatum [32], ferulic acid [145]

Free thiols (+)-Catechin [46] 
TABLE 2: Continued.

Aggressive factors

Glutathione peroxidase (GPx)

Glutathione reductase (GRed)

Reduced glutathione (GSH)

Glutathione S-transferase (GST)

(-)-Epicatechin 3-O-gallate and (-)-epigallocatechin 3-O-gallate [81], Artemisia campestris [55], Boerhaavia diffusa [172], breviscapine [51], Coccinia indica [111], coconut water [61], curcumin [108], d-pinitol [2], ellagic acid [37], Flammulina velutipes [53], Paeonia suffruticosa [40], Pleurotus eryngii [57], Spirogyra neglecta [176]

Coccinia indica [111], d-pinitol [2]

(-)-Epicatechin 3-O-gallate and (-)-epigallocatechin 3-O-gallate [81], Anogeissus acuminata [56], Artemisia campestris [55], Bacopa monnieri [30], Boerhaavia diffusa [172], Cornus officinalis [47], diosgenin [62], d-pinitol [2], ellagic acid [37], Hypericum perforatum [31], Paeonia emodi [63], Punica granatum [32], secoisolariciresinol diglucoside [88], berberine [44], ferulic acid [145]

Coccinia indica [111], d-pinitol [2]

(-)-Epicatechin 3-O-gallate and (-)-epigallocatechin 3-O-gallate [81], Abelmoschus manihot [156], Artemisia campestris [55], Bacopa monnieri [30], berberine [95] (human), Boerhaavia diffusa [172], breviscapine [51], crocin [73], Superoxide dismutase (SOD) curcumin [108], curcumin [41], diosgenin [62], d-pinitol [2], ellagic acid [37], emodin [94], Flammulina velutipes [53], Hypericum perforatum [31], isorhamnetin [87], Paeonia emodi [63], Paeonia suffruticosa [40], Physalis angulata [64], Pleurotus eryngii [57], Punica granatum [32], secoisolariciresinol diglucoside [88], berberine [44], ferulic acid [145]

Advanced glycation end-products (AGEs)

(-)-Epicatechin 3-O-gallate and (-)-epigallocatechin 3-O-gallate [81], Bacopa monnieri [30], berberine [118], coconut water [61], Cordyceps militaris [114],

AGEs Cornus officinalis [47], curcumin [41], diosgenin [62], d-pinitol [2], Paeonia emodi [63], Paeonia suffruticosa [40], Panax quinquefolium [83], ferulic acid [145]

Receptor for AGEs (RAGE)

Berberine [118], Panax quinquefolium [83]

\author{
AMPK \\ AMPK/mTOR \\ AMPK/mTOR/ULK1 \\ HO-1 \\ Hypoxic stress: SIRT-1, Foxo3/Bnip3/Hif1a \\ MAPKs (p38/, JNK, ERK 1/2), NF- $\kappa$ B \\ miR-141-3p/PTEN/Akt/mTOR \\ miR-383-5p \\ miRNA-18a-5p \\ Oxidative stress (HO-1, p47phox) \\ PI3K/AKT \\ Pim1/p21/mTOR \\ Promyelocytic leukemia zinc finger protein \\ SIRT-1 \\ SIRT1/NF- $\kappa$ B \\ $\beta$-Arrestin-1
}

Akt

Angiotensin II (AT2)

Inducible nitric oxide synthase (iNOS)

\section{Autophagy}

Astragaloside IV [103], berberine [136]

Cyclocarya paliurus - triterpenic acids-enriched fraction [142], astragaloside IV [103], berberine [136]

Mangiferin [139]

Celastrol [170]

Resveratrol [144]

Ferulic acid [145]

Triptolide [141]

Resveratrol [168]

Resveratrol [133]

Vigna angularis [146]

Curcumin [132], berberine [44]

Hispidulin [137]

Tangshen Formula [123]

Abelmoschus manihot [143]

astragaloside IV [131]

Tripterygium glycosides [138]

\section{Pathways}

(-)-Epigallocatechin-3-gallate [155], Abelmoschus manihot [156], emodin [94]

(-)-Epigallocatechin-3-gallate [155]

(-)-Epicatechin 3-O-gallate and (-)-epigallocatechin 3-O-gallate [81], Cornus officinalis [47], curcumin [108], curcumin analogue (B06) [66], Hypericum perforatum [31], moringa isothiocyanate [42], secoisolariciresinol diglucoside [88] 
TABle 2: Continued.

\author{
Aggressive factors \\ Cyclooxygenase 2 (COX-2) \\ Endoplasmic reticulum stress (ER stress) \\ c-Jun NH2-terminal kinase (JNK) \\ Extracellular regulated protein kinases (ERK1/2) \\ Glycogen synthase kinase $3 \beta$ (GSK-3 $\beta$ )
}

Nuclear factor $\kappa \mathrm{B}(\mathrm{NF}-\kappa \mathrm{B})$

Nucleotide binding and oligomerization

domain-like receptor family pyrin

domain-containing 3 (NLRP3) inflammasome

Smad (TGF- $\beta /$ Smad signaling)

Sterol regulatory element-binding protein (SREBP)

Transforming/tumor growth factor $\beta$ (TGF- $\beta$ )

p38-mitogen-activated protein kinase (p38MAPK)

Phosphatidylinositol 3-kinase (PI3K)

Proteinkinase C (PKC)

JNK (JNK-NF- $\kappa$ B signaling)

Insulin receptor (IR) and insulin

receptor substrate (IRS)

Glucose transporter 1 and 4 (GLUT1, GLUT4)

$\mathrm{G}$ protein-coupled receptor kinase

(GRK)2, GRK3, GRK6

$\mathrm{PGE} 2 / \mathrm{EP} 1 / \mathrm{G} \alpha \mathrm{q} / \mathrm{Ca}^{2+}$

cAMP response element-binding protein (CREB)

Cyclic adenosine monophosphate (cAMP)
(-)-Epicatechin 3-O-gallate and (-)-epigallocatechin 3-O-gallate [81], Cornus officinalis [47], curcumin analogue (B06) [66], Hypericum perforatum [31] Abelmoschus manihot [104], astragaloside IV [15], astragaloside IV [103] Abelmoschus manihot [104]

(-)-Epigallocatechin-3-gallate [155]

Emodin [94]

(-)-Epicatechin 3-O-gallate and (-)-epigallocatechin 3-O-gallate [81], berberine [85], Coreopsis tinctoria [72], Cornus officinalis [47], crocin [73], curcumin [79], curcumin analogue (B06) [66], d-pinitol [2], ellagic acid [37], Fragaria $\times$ ananassa [89], Hypericum perforatum [31], isorhamnetin [87], Morus alba [77], Paederia foetida [78], Paeonia lactiflora [120], Panax quinquefolium [82], Prunella vulgaris [84], puerarin [76], Zea mays anthocyanins [70], secoisolariciresinol diglucoside [88], Shen-Yan-Fang-Shuai Formula [80], Spirogyra neglecta [176], astragaloside IV [131], Abelmoschus manihot [143], ferulic acid [145]

Dihydroquercetin [48]

Berberine [85], cocoa enriched with polyphenols [110], Coreopsis tinctoria [72], Hydrangea paniculata [59], Prunella vulgaris [84], Zea mays anthocyanins [70], Tangke decoction [116]

Cornus officinalis [47], curcumin [108], Fragaria $\times$ ananassa [89], myricetin [157]

(-)-Epicatechin 3-O-gallate and (-)-epigallocatechin 3-O-gallate [81], Abelmoschus manihot [104], Abelmoschus manihot [156], berberine [121], berberine [85], berberine [117], berberine [118], breviscapine [51], breviscapine [52], cocoa enriched with polyphenols [110], Cordyceps militaris [114], Coreopsis tinctoria [72], curcumin [108], curcumin [79], curcumin analogue (B06) [66], Dioscorea bulbifera [96] (human), ellagic acid [37], Hypericum perforatum [31], isorhamnetin [87], moringa isothiocyanate [42], myricetin [157], Paeonia lactiflora [120], Paeonia suffruticosa [109], Panax quinquefolium [82], Prunella vulgaris [84], Psoralea corylifolia [115], Zea mays anthocyanins [70], Qidan Dihuang [119], rhein [113], Shen-Yan-Fang-Shuai Formula [80], Tangke decoction [116]

(-)-Epigallocatechin-3-gallate [155], Abelmoschus manihot [156], Danhong [154], emodin [153]

(-)-Epigallocatechin-3-gallate [155], Huangqi decoction [36]

Berberine [118], breviscapine [51], breviscapine [52], Cladophora glomerata [34] Astragaloside IV [15], curcumin analogue (B06) [66]

Huangqi decoction [36]

Huangqi decoction [36]

Berberine [121]

Berberine [122]

Emodin [153]

Berberine [158], berberine [121], emodin [153]

Mediators

Matrix metalloproteinase (MMP)

MMP-1

MMP-9 and MMP-9/TIMP-1 ratio

Tissue inhibitor of MMPs- (TIMP-) 2 and MMP-2/TIMP-2 ratio
Zea mays anthocyanins [70]

Berberine [117], puerarin [60], rhein [127]

Berberine [117], Zea mays anthocyanins [70], 
TABle 2: Continued.

\begin{tabular}{|c|c|}
\hline \multicolumn{2}{|l|}{ Aggressive factors } \\
\hline \multicolumn{2}{|r|}{ Cytokines } \\
\hline IL-1 (interleukin-1) & $\begin{array}{l}\text { Abelmoschus manihot [104], berberine [85], crocin [73], curcumin [79], } \\
\text { curcumin analogue (B06) [66], dihydroquercetin [48], d-pinitol [2], ellagic acid } \\
\text { [37], Hypericum perforatum [31], isorhamnetin [87], moringa isothiocyanate } \\
\text { [42], Morus alba [77], Paederia foetida [78], Paeonia lactiflora [120], celastrol } \\
\text { [170], resveratrol [144], ferulic acid [145] }\end{array}$ \\
\hline IL-1 receptor (IL-1R) & Morus alba $[77]$ \\
\hline IL-10 & Crocin [73], resveratrol [144] \\
\hline IL-12 & Curcumin analogue (B06) [66] \\
\hline IL-2 & Abelmoschus manihot [104] \\
\hline IL-6 & $\begin{array}{l}\text { Abelmoschus manihot [104], curcumin analogue (B06) [66], Dioscorea bulbifera } \\
\text { [96] (human), d-pinitol [2], ellagic acid [37], emodin [94], Fragaria } \times \text { ananassa } \\
\text { [89], isorhamnetin [87], moringa isothiocyanate [42], Morus alba [77], Paederia } \\
\text { foetida [78], Paeonia suffruticosa [40], Salacia chinensis [102] (human), celastrol } \\
\text { [170], resveratrol [144], Abelmoschus manihot [143], ferulic acid [145] }\end{array}$ \\
\hline IL-8 & Crocin $[73]$ \\
\hline Interferon- $\gamma$ & Morus alba [77] \\
\hline Tumor necrosis factor- $\alpha$ (TNF- $\alpha)$ & $\begin{array}{l}\text { Abelmoschus manihot [104], Abelmoschus manihot [156], Allium sativum [28], } \\
\text { berberine [85], crocin [73], curcumin [79], curcumin analogue (B06) [66], } \\
\text { d-pinitol [2], ellagic acid [37], emodin [94], Fragaria } \times \text { ananassa [89], Hypericum } \\
\text { perforatum [31], isorhamnetin [87], Morus alba [77], Paederia foetida [78], } \\
\text { Paeonia lactiflora [120], secoisolariciresinol diglucoside [88], Shen-Yan-Fang- } \\
\text { Shuai Formula [80], silymarin [65] (human), astragaloside IV [103], celastrol } \\
\text { [170], resveratrol [144], Abelmoschus manihot [143], ferulic acid [145] }\end{array}$ \\
\hline \multicolumn{2}{|r|}{ Chemokines } \\
\hline $\begin{array}{l}\text { Monocyte chemoattractant/chemotactic } \\
\text { protein-1 (MCP-1) }\end{array}$ & $\begin{array}{l}\text { berberine [85], breviscapine [51], Coreopsis tinctoria [72], curcumin [79], } \\
\text { curcumin analogue (B06) [66], Hypericum perforatum [31], moringa } \\
\text { isothiocyanate [42], Paeonia suffruticosa [40], Prunella vulgaris [84], Zea mays } \\
\text { anthocyanins [70], Shen-Yan-Fang-Shuai Formula [80], astragaloside IV [103], } \\
\text { ferulic acid [145] }\end{array}$ \\
\hline \multicolumn{2}{|r|}{ Adhesion molecules } \\
\hline Intercellular adhesion molecule-1 (ICAM-1) & $\begin{array}{l}\text { Breviscapine [51], curcumin [79], emodin [94], Hypericum perforatum [31], } \\
\text { isorhamnetin [87], Paeonia lactiflora [120], Prunella vulgaris [84], Zea mays } \\
\text { anthocyanins [70], ferulic acid [145] }\end{array}$ \\
\hline Vascular cell adhesion molecule-1 (VCAM-1) & Berberine [95], ferulic acid [145] \\
\hline \multicolumn{2}{|r|}{ Growth factors } \\
\hline Vascular endothelial growth factor (VEGF) & Myricetin [157], Panax quinquefolium [82] \\
\hline Connective tissue growth factor (CTGF) & Emodin [153], Prunella vulgaris [84], Zea mays anthocyanins [70] \\
\hline \multicolumn{2}{|r|}{ Cellular infiltration } \\
\hline Macrophage infiltration & Breviscapine [51], curcumin [79], curcumin analogue (B06) [66] \\
\hline Macrophage migration & Paeonia suffruticosa $[40]$ \\
\hline Myeloperoxidase (MPO) & $\begin{array}{l}\text { (-)-Epicatechin 3-O-gallate and (-)-epigallocatechin 3-O-gallate [81], } \\
\text { diosgenin [62] }\end{array}$ \\
\hline \multicolumn{2}{|r|}{ Diabetic nephropathy (DN) } \\
\hline \multicolumn{2}{|r|}{ Apoptosis } \\
\hline Apoptosis & $\begin{array}{l}\text { Astilbin [140], astragaloside IV [15], curcumin [41], Psoralea corylifolia [115], } \\
\text { quercetin [49], secoisolariciresinol diglucoside [88], silybin [43], berberine [45], } \\
\text { berberine [44], berberine [136], tripterygium glycosides [138], celastrol [170], } \\
\text { curcumin [132], resveratrol [133], astragaloside IV [103], Cyclocarya } \\
\text { paliurus - triterpenic acids-enriched fraction [142], ferulic acid [145] }\end{array}$ \\
\hline HIF- $1 \alpha / \mathrm{PI} 3 \mathrm{~K} / \mathrm{Akt}$ & Berberine $[45]$ \\
\hline Bcl-2-associated X protein (BAX) & Astilbin [140], emodin [94], berberine [45], berberine [44] \\
\hline
\end{tabular}


TABLE 2: Continued.

\begin{tabular}{ll}
\hline Aggressive factors & \\
\hline B-cell lymphoma 2 protein (Bcl-2) & $\begin{array}{l}\text { Astilbin [140], emodin [94], Psoralea corylifolia [115], secoisolariciresinol } \\
\text { diglucoside [88] } \\
\text { Psoralea corylifolia [115] } \\
\text { Bcl-2-associated death promoter (Bad) } \\
\text { Caspase-9 }\end{array}$ Astragaloside IV [103] \\
Caspase-12 & Astilbin [140], astragaloside IV [15], emodin [94], Hypericum perforatum [31], \\
& berberine [45], berberine [44], curcumin [132], resveratrol [133], resveratrol \\
Caspase-3 & [168], astragaloside IV [103], Cyclocarya paliurus-triterpenic acids-enriched \\
& fraction [142], ferulic acid [145] \\
Caspase-1 & Dihydroquercetin [48] \\
Poly (ADP-ribose) polymerase (PARP) & Psoralea corylifolia [115] \\
Survivin (antiapoptotic marker) & Secoisolariciresinol diglucoside [88] \\
C/EBP homologous protein (CHOP) & Astragaloside IV [15] [103] \\
\hline
\end{tabular}

Collagen 1

Glomerulosclerosis (GS), fibrosis, extracellular matrix (ECM) expansion

Collagen 3

Berberine [85]

Tangshen Formula [123]

Abelmoschus manihot [104], berberine [121], berberine [85], berberine [117], cocoa enriched with polyphenols [110], Cordyceps militaris [114], Coreopsis

Collagen 4 tinctoria [72], dihydroquercetin [48], Hydrangea paniculata [59], Hypericum perforatum [31], myricetin [157], Paeonia lactiflora [120], Panax quinquefolium [82], Prunella vulgaris [84], Psoralea corylifolia [115], Zea mays anthocyanins [70], Shen-Yan-Fang-Shuai Formula [80], triptolide [133], astragaloside IV [131]

(-)-Epicatechin 3-O-gallate and (-)-epigallocatechin 3-O-gallate [81], (+)-catechin [46], berberine [85], berberine [117], Coccinia indica [111], cocoa enriched with polyphenols [110], Coreopsis tinctoria [72], dihydroquercetin [48],

Fibronectin ellagic acid [37], emodin [153], Hydrangea paniculata [59], Hypericum perforatum [31], myricetin [157], Paeonia suffruticosa [109], Panax quinquefolium [82], Psoralea corylifolia [115], rhein [113], Shen-Yan-Fang-Shuai Formula [80], triptolide [133], astragaloside IV [131]

Laminin Coccinia indica [111]

Endothelial dysfunction

Salacia chinensis [102] $(\mathrm{H})$

Endothelial dysfunction

(+)-Catechin [46], Panax quinquefolium [82]

Endothelin-1

Salacia chinensis [102] (H)

Homocysteine

Podocytes, foot processes, slit diaphragms

Podocyte apoptosis

Podocyte foot processes effacement

Podocytes CD2-associated protein

(markers of slit diaphragms reduced by HG)

Podocyte slit diaphragm proteins

(nephrin, podocin, and synaptopodin)

Podocin

Nephrin

Synaptopodin

Desmin

Caveolin-1, $\beta$-catenin Integrin-linked kinase
Silybin [43], celastrol [170], resveratrol [168]

Curcumin [130], puerarin [60]

Crocin [73]

Puerarin [60]

Crocin [73], puerarin [60], hispidulin [137], berberine [136], tripterygium glycosides [138], astragaloside IV [103]

Crocin [73], Paeonia lactiflora [120], puerarin [60], mangiferin [136], hispidulin [137], berberine [136], tripterygium glycosides [138], celastrol [170], resveratrol [168], astragaloside IV [103]

Curcumin [108]

Curcumin [108]

Epithelial-mesenchymal transdifferentiation (EMT)

Curcumin [130]

Rhein [127] 
TABLE 2: Continued.

\begin{tabular}{ll}
\hline Aggressive factors & \\
\hline Vimentin & Curcumin [108] \\
\hline & \multicolumn{1}{c}{ Protectors } \\
Akt & Astilbin [140], Huangqi decoction [36] \\
Nuclear factor erythroid-derived 2 (Nrf2) & Hydrangea paniculata [59], moringa isothiocyanate [42], berberine [44] \\
NAD(P)H:Quinone oxidoreductase 1 (NOX1) & Moringa isothiocyanate [42] \\
Glutamate cysteine ligase catalytic subunit (GCLC) & Moringa isothiocyanate [42] \\
$5^{\prime}$ adenosine monophosphate-activated & Cocoa enriched with polyphenols [110], Coreopsis tinctoria [72] \\
protein kinase (AMPK) & Puerarin [76] \\
Sirtuin 1 (SIRT1) & Abelmoschus manihot [104], myricetin [157] \\
Peroxisome proliferator-activated receptor- $\alpha$ (PPAR- $\alpha)$ & Abelmoschus manihot [104], Danhong [154], emodin [153], Fragaria $\times$ ananassa \\
Peroxisome proliferator-activated receptor- $\gamma(\mathrm{PPAR}-\gamma)$ & [89], Hypericum perforatum [31] \\
$\begin{array}{l}\text { E prostanoid receptor 4/protein G } \alpha \text { s/adenylate } \\
\text { cyclase/cyclic adenosine monophosphate }\end{array}$ & Berberine [158] \\
(EP4/G $\alpha$ s/AC/cAMP) & \\
\hline
\end{tabular}

oxygenase-1) and of hypoxia-inducible factor $1 \alpha$. Both these factors reduce HG-induced apoptosis, particularly when associated with oxidative [44] and hypoxic injury [45], respectively.

3.2.2. In Vivo Studies-Actually Nephroprotective Mechanisms. Many studies have pointed out the antioxidant properties of the investigated herbal products (catechin [46], Cornus officinalis [47], dihydroquercetin [48], etc.-see Table 2), translated in their ability to diminish the burden of ROS (quercetin [49], etc.), reactive nitrogen species (tocotrienol [50], etc.), and lipid peroxidation products (breviscapine [51, 52], Flammulina velutipes [53], Hibiscus sabdariffa [54], and many more-see Table 2). Beside decreasing malondialdehyde, Artemisia campestris also lowered the level of nitric oxide (NO) and advanced oxidation protein products [55]. Most of these plant-derived products are also able to increase the antioxidant capacity (Anogeissus acuminata [56], Pleurotus eryngii [57], Punica granatum [32], etc.-see Table 2 for a complete list).

Suppressing the oxidative stress prevents inflammation and cell death. Therefore, the antioxidant effect maintains cells alive (as silybin does with the HG-damaged podocytes [43]) and decreases inflammation (garlic reduced not only the burden of oxidative stress but also the level of TNF- $\alpha$ [28]). The antioxidant armamentarium of some phytoagents includes the activation of Nrf2, in conjunction with ameliorating mitochondrial dysfunction, dampening the inflammatory response, and bolstering the function of antioxidant enzymes (curcumin [58]) or with suppressing TGF/Smad signaling and the glomerular accumulation of fibronectin and collagen 4 (Hydrangea paniculata [59]). Puerarin (from Pueraria lobata) prevents podocyte foot process effacement and boosts the expression of podocyte slit diaphragm proteins such as nephrin and podocin, beside attenuating oxidative and nitrosative stress, and one of their consequences, the activation of MMP-9 [60].
Apart from the antioxidant capacity, some herbal products have also demonstrated an ability to decrease the production of AGEs (Bacopa monnieri [30], coconut water [61], diosgenin [62], Paeonia emodi [63], Physalis angulata [64], etc.-see Table 2). A cyclitol from soybean, d-pinitol, has been shown to decrease both AGEs and inflammationpromoting factors [2].

3.2.3. Human Studies-Actually Nephroprotective Mechanisms. A study done on human subjects has proved the ability of silymarin to boost the antiproteinuric activity of reninangiotensin system (RAS) inhibitors [65].

3.3. Herbal Products with Anti-Inflammatory Activity. Inflammation is a key pathophysiologic component in the genesis of DN [66-68]. The involvement of microinflammation in the pathogenesis of DN justifies the interest for antiinflammatory herbal products in preventing $\mathrm{DN}$-this concept may be correlated with the thousands years old employment of "heat-clearing" herbs for the treatment of DN in Traditional Chinese Medicine [69]. Herbal products may decrease inflammation by multiple ways, such as lowering the level of proinflammatory cytokines (TNF- $\alpha$, IL- $1 \beta$, IL-6, IL-12, etc.), decreasing the factors promoting inflammatory cells infiltration (chemokines such as MCP-1 and adhesion molecules including ICAM-1 and vascular cell adhesion molecule-1), and modulating the inflammatory pathways and/or the activity of transcription factors.

3.3.1. In Vitro Studies-Potentially Nephroprotective Mechanisms. Purple corn (Zea mays) anthocyanins hindered the interaction between NF- $\kappa$ B and canonical TGF- $\beta 1$ (i.e., Smad) pathways. Consequently, it thwarted ECM expansion by both promoting ECM degradation and reducing new ECM formation as a result of TGF- $\beta 1$ signaling inhibition blocking the induction of ICAM-1 and MCP-1 (responsible for connective tissue growth factor expression) and the 
secretion of collagen 4 (essential for mesangial hyperplasia). ECM dissolution was the consequence of higher membrane type-1 MMP and lower tissue inhibitor of MMP- (TIMP-) 2 expression [70].

AMPK ( $5^{\prime}$ adenosine monophosphate-activated protein kinase) acts as an energy sensor and undermines TGF$\beta 1 /$ Smad pathway by hindering Smad 4 translocation into the nucleus, thereby impeding ECM accumulation [71]. Coreopsis tinctoria and its main component, the chalconoid marein, blocked both TGF- $\beta 1 /$ Smad (by means of p-AMPK) and NF- $\kappa \mathrm{B}$ pathways, and consequently attenuated inflammation, mesangial cell proliferation, and fibrogenesis [72].

Crocin, the carotenoid from Crocus sativus responsible for its saffron color, has also been proven a podocytefriendly substance. It is able to foster the integrity of glomerular filtration barrier, demonstrated by higher levels of slit diaphragms markers: nephrin, podocin, and CD2associated protein. Moreover, it diminishes oxidative stress and the proinflammatory response of the podocytes (by NF- $\kappa$ B inactivation) [73].

SIRT1 (Sirtuin 1) suppresses the activity of NF- $\kappa$ B by deacetylating its RelA/p65 subunit $[74,75]$. Puerarin from the roots of Pueraria lobata increased the level and activity of SIRT1 protein in podocytes, followed by enhanced SIRT1mediated deacetylation (hence, inactivation) of NF- $\kappa \mathrm{B}$ and reduced NOX4 expression [76].

3.3.2. In Vivo Studies-Actually Nephroprotective Mechanisms. Downregulating the activity of $N F-\kappa B$ dependent pathways is one of the most explored anti-inflammatory renoprotective mechanism of the herbal products. In most studies, NF- $\kappa \mathrm{B}$ inactivation is accompanied by lower levels of proinflammatory factors, including various combinations of proinflammatory cytokines: IL-1 (and IL-1 receptor), IL-6, interferon- $\gamma$, and TNF- $\alpha$ (Morus alba [77]), TNF- $\alpha$, IL- $1 \beta$, and IL-6 (Paederia foetida [78], d-pinitol from soybean [2]).

Other studies demonstrated, aside from NF- $\kappa B$ inactivation and lower levels of proinflammatory cytokines, a decrease in the expression of chemokines (mainly MCP-1) and of the factors spurring sclerosis/fibrosis (especially TGF- $\beta 1$ ), thereby warding off macrophage infiltration (curcumin [79]) and suppressing the production of collagen 4 and fibronectin, and hence ECM expansion and glomerulosclerosis (ellagic acid [37], Shen-Yan-Fang-Shuai Formula [80]). Added to these effects, the inhibition cyclooxygenase2 and inducible NO synthase boosted the anti-inflammatory potency of green tea polyphenols [81], of Hypericum perforatum [31], and of curcumin analogue B06 (in the latter case probably by means of blocking the JNK (c-Jun N-terminal kinase)/NF- $\kappa B$ signaling [66]). Suppressing the production of vasoactive factors (vascular endothelial growth factor, endothelin-1) resulted in an all-encompassing protective spectrum for North American ginseng, which has been shown to decrease inflammation, fibrosis, and mesangial expansion, to improve the oxidative and metabolic status [82], and to suppress AGEs generation [83]. Other members of the Panax genus have also been shown to prevent diabetic glomerular lesions (P. notoginseng [35]). The aptitude to block both inflammation and fibrosis is also manifested by plant extracts that conjointly inhibit NF- $\kappa \mathrm{B}$ and canonical TGF- $\beta 1$ (i.e. Smad) pathways (Prunella vulgaris [84]), berberine [85]). Arctigenin from Fructus arctii also can improve the viability and function of the podocytes conjointly with attenuating the NF- $\kappa \mathrm{B}$-mediated inflammatory effects due to protein phosphatase $2 \mathrm{~A}$-mediated decrease in p65 NF$\kappa \mathrm{B}$ activating phosphorylation [86].

Several herbal products hampered the production of ROS conjointly to inhibiting $N F-\kappa B$ dependent inflammatory pathways (puerarin [76]) and the production/activation of various proinflammatory cytokines (Paederia foetida [78]), adhesion molecules, and fibrosis promoting cytokines (isorhamnetin, present in onions, sea buckthorn, and various other medicinal plants [87]). Secoisolariciresinol diglucoside (from Linum usitatissimum) is able not only to diminish the inflammatory and oxidative aggression on the kidney cells, but also to prevent their death, as reflected by higher levels of antiapoptotic markers survivin and B-cell lymphoma-2 (Bcl-2) [88].

Hypericum perforatum seems to exert an all-embracing nephroprotective action, the anti-inflammatory and antioxidant effects being complemented by antifibrosing and antiapoptotic activity (inhibition of caspase- 3 and cytochrome c), and enhanced expression of PPAR (peroxisome proliferatoractivated receptor) $-\gamma$ [31]. Strawberry (Fragaria $\times$ ananassa) extracts also combine the PPAR- $\gamma$ pathway activating effect with an anti-inflammatory one, associated with suppression of several fatty acid synthesis genes and of the sterol regulatory element-binding protein $(S R E B P)$ transcription factor [89], one of the inducers of TGF- $\beta 1$ that may be activated by a HG-milieu [90]. The inactivation of both SREBP and NF- $\kappa \mathrm{B}$ pathways, coupled with antioxidant activity and decreased production of AGEs, was proven for the fruit of Cornus officinalis, having morroniside, loganin, and 7-O-galloyl-D-sedoheptulose as the main active compounds [47].

The NLRP3 (nucleotide binding and oligomerization domain-like receptor family pyrin domain-containing 3) inflammasome triggers inflammatory events by means of activating caspase- 1 , which leads to both proinflammatory cytokines (such as IL-1 $\beta$ and IL-18) activation and to pyroptosis, a type of inflammatory cell death [91]. The dihydroflavone dihydroquercetin blocked the activation of NLRP3 inflammasome, besides reducing cell proliferation, ROS generation, and the expression of renal fibrosis-associated proteins [48].

The activation of the GSK-3 $\beta$ pathway is known to ameliorate diabetes-induced kidney injury. GSK- $3 \beta$ inactivation by $P I 3 K / A k t$-mediated phosphorylation increases protein synthesis associated with diabetic glomerular hypertrophy and sclerosis $[12,92]$. Akt (protein kinase B) is involved in metabolism (particularly glucose metabolism), growth, proliferation, and survival/apoptosis. PI3K is a key regulator of the multi-step process controlling Akt activation [93]. Emodin, the main active component of rhubarb (Rheum officinale), exerted its anti-inflammatory, antiapoptotic [decrease in B-cell lymphoma 2-associated X protein (Bax) and caspase- 3 expression], and antioxidative activities by triggering PI3K/Akt/GSK-3 $\beta$ signaling pathway [94]. However, it is presently not clear whether PI3K/Akt induction should be 
considered protective or deleterious for the diabetic kidney, as among the phytoagents useful for preventing DN some turn this pathway on, while others turn it off [13].

\subsubsection{Human Studies-Actually Nephroprotective Mechanisms.} In one of the very few human trials, berberine lowered the level of vascular cell adhesion molecule- 1 and C-reactive protein, as well as the urinary markers of kidney injury. It also favorably tipped the oxidative stress balance (decreasing lipid peroxidation and nucleic acid oxidation and augmenting the total-antioxidant capacity) and improved renal hemodynamics. Hence, berberine may emerge as a nephroprotective agent able to complement standard hypotensive and hypoglycemic treatment [95]. In another trial on human subjects, Dioscorea bulbifera provided a better control than fosinopril of blood pressure, metabolic, biologic, and inflammatory parameters in patients with $\mathrm{DN}$ [96].

3.4. Preventing Endothelial Dysfunction. The nephroprotective effect of some herbal products includes the prevention of endothelial dysfunction, an attribute of DN epitomized by the imbalance between endothelium-derived vasodilators and vasoconstrictors, the former preventing thrombosis and proliferation and the latter promoting atheroma formation [14]. Endothelial dysfunction is reflected by biomarkers such as endothelin 1 [97], homocysteine [98], and IL-6 (a proinflammatory cytokine) [99]. Induced by angiotensin II [100], oxidative stress, inflammation, and hypoxia [46], endothelin 1 promotes afferent and efferent arterioloconstriction with declining glomerular filtration rate [101].

3.4.1. In Vivo Studies-Actually Nephroprotective Mechanisms. A component of green tea, $(+)$-catechin, has been proven to mitigate endothelial dysfunction, as reflected by diminished endothelin 1 levels, beside exerting antifibrosing and antioxidant activity [46].

3.4.2. Human Studies-Actually Nephroprotective Mechanisms. A study on stable diabetic CKD patients revealed the ability of Salacia chinensis to forestall endothelial dysfunction, demonstrated by a decrease in the levels of homocysteine and IL-6 ([102]).

3.5. Preventing ER stress. Endoplasmic reticulum (ER) stress is an important link in the pathophysiological chain leading to $\mathrm{DN}$, consisting in improperly folded proteins piling up in the ER lumen and consequently unchaining the unfolded protein response (UPR), which may lead to either cell protection or cell death by apoptosis $[15,16]$. SERCA dysfunction is an important inductor of ER stress [103].

3.5.1. In Vivo Studies-Actually Nephroprotective Mechanisms. Abelmoschus manihot extract (Huangkui capsule) attenuates ER stress in correlation with reduced JNK activation [104]. Astragaloside IV (derived from Astragalus membranaceus) dampens ER stress and, consequently, podocyte apoptosis [15] by means of SERCA2 [103].

3.6. Herbal Products with Antifibrosing/Antiproliferative Activity. TGF- $\beta 1$ has a crucial role in the progression of DN [105] leading to EMT [106], ECM expansion, collagen biosynthesis, and renal cell growth [10]. The result is glomerular and whole kidney hypertrophy [107] that, together with thickening of the glomerular basement membrane, are emblematic for DN [108].

3.6.1. In Vitro Studies-Potentially Nephroprotective Mechanisms. Paeoniflorin, pentagalloylglucose, and paeonol from the root bark of Paeonia suffruticosa have been shown to suppress TGF- $\beta 1$ signaling, thereby reducing the levels of fibronectin ([109]). Purple corn anthocyanins too interfere with TGF- $\beta 1 /$ Smad pathway (and NF- $\kappa$ B signaling), beside reducing the expression of connective tissue growth factor and collagen 4 and accelerating ECM dissolution [70].

3.6.2. In Vivo Studies-Potentially Nephroprotective Mechanisms. A study performed on spontaneously hypertensive rats with streptozotocin-induced diabetes has shown that activating phosphorylation of AMPK by cocoa enriched with polyphenols may suppress TGF- $\beta 1 /$ Smad pathway (by blocking Smad2 phosphorylation), resulting in diminished levels of TGF- $\beta 1$, collagen 4 , and fibronectin ([110]).

3.6.3. In Vivo Studies-Actually Nephroprotective Mechanisms. Tea catechins, a class of flavonoids, have been shown to decrease interstitial fibrosis [3]. Coccinia indica could prevent the accumulation of ECM by decreasing fibronectin and laminin in association with antioxidant activity [111].

Arguably the most efficient mechanism for preventing fibrosis/sclerosis is downregulation of its key promoter, TGF- $\beta 1$, and of the related signaling pathways. Activation of TGF- $\beta 1$ type I receptors leads to Smad 2 and Smad3 phosphorylation, followed by binding to $\operatorname{Smad} 4$, the resultant oligomeric complex translocating into the nucleus, where it regulates the transcription of key proteins responsible for renal fibrogenesis [112]. Abating TGF- $\beta 1$ expression is typically associated with lower levels of fibronectin (rhein [113]), collagen (Cordyceps militaris [114]), or both, sometimes accompanied by antiapoptotic effects, as is the case for Psoralea corylifolia seed extract and two of its major components, isopsoralen and psoralen. These phytoagents downregulated proapoptotic proteins [such as cleaved poly (ADP-ribose) polymerase and $\mathrm{Bcl}-2$-associated death promoter (Bad)] and buttressed cell viability, as indicated by higher levels of survival markers p-Bad (ser112) and B-cell lymphoma(Bcl-) 2 [115].

Declining levels of TGF- $\beta 1$, collagen 4 , and fibronectin may be correlated with $T G F-\beta 1 /$ Smad pathway mitigation (Tangke decoction [116], Hydrangea paniculata [59]) by means of activating phosphorylation of AMPK leading to reduced phosphorylation of Smad2 via diminished NOX4 expression (berberine [117]). The shrinking levels of TGF$\beta 1$ may also be coupled with reduced activity and expression of $P K C$, associated with antioxidant activity (breviscapine [52]) and anti-inflammatory effect (reduced macrophage infiltration) (again breviscapine [51]), and with suppression of AGEs/RAGE/PKC- $\beta /$ TGF- $\beta 1$ signaling pathway (berberine [118]). The decrease in TGF- $\beta 1$ level may also be associated with inhibition of the RAS and of the myofibroblast proliferation (low $\alpha$-smooth muscle actin) (Qidan 
Dihuang decoction [119]) or with anti-inflammatory action reflected in lower levels of inflammatory promoters (Paeonia lactiflora [120]).

Modulation of $G$ protein/AC (adenylyl cyclase)/cAMP (cyclic adenosine monophosphate) signaling pathway by means of altering $G$ protein-coupled receptor kinases may be connected with dwindling TGF- $\beta 1$ and collagen 4 expression in the case of berberine [121]. Berberine also interfered with prostaglandin $\mathrm{E}_{2} /$ E prostanoid receptor $(E P) 1 / G \alpha q / \mathrm{Ca}^{2+}$ pathway, its depressing effects being associated with a decline in mesangial cells proliferation [122]. By blocking the SREBP, which is known to upregulate TGF- $\beta 1$ [90], curcumin was linked to diminished expression of TGF- $\beta 1$, EMT suppression (diminished vimentin), and podocyte protection (low desmin and high synaptopodin and connexin 43) [108]. Tangshen Formula combines the antifibrosing effect with an autophagy-inducing one, achieved by inhibiting the pathway leading from promyelocytic leukemia zinc finger protein $(P L Z F)$ activation to a decline in autophagy and autophagy-induced collagen 3 dissolution. Hence, the effect of Tangshen Formula is more autophagy and less collagen 3 accumulation, and consequently less tubulointerstitial ECM deposition [123].

3.6.4. Human Studies-Actually Nephroprotective Mechanisms. A multicenter randomized, double-blind, placebo-controlled trial on the efficacy of Tangshen Formula in DN yielded promising results: after a mere 6 months, the improvements in proteinuria and estimated glomerular filtration rate (eGFR) were significant [124], as was the decrease in livertype fatty acid binding protein [125], a biomarker correlated with the severity and prognosis of DN [126]. Longer duration interventions are clearly needed for defining the role of this formula in preventing $\mathrm{DN}$.

3.7. Herbal Products Blocking Epithelial-Mesenchymal Transdifferentiation. Fibrosis and ECM synthesis are bolstered by EMT, in which the epithelial cells trade their epithelial features (reflected by proteins such as E-cadherin) for mesenchymal ones (correlated with the expression of $\alpha$ smooth muscle actin and vimentin, among others) [4]. TGF- $\beta 1$ is one of the main inducers of EMT by means of both the canonical pathway (TGF- $\beta / \mathrm{Smad} 2 / 3$ ) and the non-canonical one (MAPK/PI3K) [106]. Consequently, all herbal products able to forestall TGF- $\beta 1$ generation are potentially apt to thwart EMT.

3.7.1. In Vitro Studies-Potentially Nephroprotective Mechanisms. Matrix metalloproteinase- (MMP-) 9 promotes EMT by degrading type IV collagen of the basement membranes (thereby facilitating the migration of the transdifferentiated epithelial cells through the altered basement membrane) and by preventing the dissolution of ECM, which furthers renal interstitial fibrosis. The overactivity of MMP-9 results from insufficient inhibition by TIMP-1. The MMP9/TIMP-1 imbalance is induced by integrin-linked kinase (ILK), a downstream mediator on a pathway driven by TGF- $\beta 1$ [127]. Rhein hampers EMT by suppressing ILK, thus correcting the excessively high MMP-9/TIMP-1 [127].
3.7.2. In Vivo Studies-Actually Nephroprotective Mechanisms. Caveolin-1 is involved in regulating cell junctions, including E-cadherin/ $\beta$-catenin complex, an adhesion complex linked to actin filaments. The $\beta$-catenin component functions as a coactivator in the $\mathrm{Wnt} / \beta$-catenin signaling pathway that accelerates the EMT of podocytes [128-130]. Curcumin inhibits caveolin-1 Tyr(14) phosphorylation which stabilizes caveolin- 1 and $\beta$-catenin, thus blocking EMT of podocytes [130]; it also suppresses the EMT of tubular cells, as reflected by lower levels of vimentin, an important marker of this process [108].

3.8. Herbal Products that Restore Autophagy. By degrading damaged cellular components, autophagy, an adaptive response of cells exposed to various environmental stresses, is essential for maintaining the structural and functional well-being of podocytes [103, 131, 132]. Consequently, its malfunction in DN leads to podocyte loss and proteinuria [133]. As it acts as a protective mechanism [134], its inactivation contributes to disease progression [135]. Nonetheless, some authors have found autophagy activation in DN-this seems to be an early, short-lived event, followed by autophagy depression in the long run [135].

3.8.1. In Vitro Studies-Potentially Nephroprotective Mechanisms. Several pathways converge in regulating autophagy and are the points of impact of the various herbal products that enhance cell viability by activating autophagy: AMPK (berberine [136]), mTOR (hispidulin [137]), PI3K/AKT (curcumin [132]), and $\beta$-arrestin-1 (tripterygium glycosides [138]). Besides increasing autophagy, celastrol also dampens inflammation, oxidative stress, and apoptosis and hence increases podocyte viability, as reflected by enhanced expression of nephrin [139]. Still, there are herbal products whose favorable action is associated with reduced autophagy, such as astilbin, a flavanonol found in several plants, including Hypericum perforatum. The beneficial effect of astilbin on HG-treated human proximal tubular epithelial cells was associated with diminished apoptosis and autophagy, resulting from Akt induction (mirrored by higher levels of p-Akt) [140]. This may be explained by autophagy activation early in the course of DN.

3.8.2. In Vivo Studies-Actually Nephroprotective Mechanisms. In vivo (or combined in vivo and in vitro) studies confirmed the ability of herbal products to act on the pathways already mentioned: AMPK (astragaloside IV [103]), mTOR (triptolide [141]), and AMPK/mTOR (triterpenic acids-enriched fraction of Cyclocarya paliurus [142], mangiferin [139]). Several other pathways have also been revealed: SIRT1 (Abelmoschus manihot [143], astragaloside IV [131], resveratrol [144]), MAPKs (ferulic acid [145]), PLZF (Tangshen Formula [123]), and miR-18a-5p/atactic telangiectasis mutation (resveratrol [133]). Some herbal products have the ability to act on the pathways driven by one or another environmental stressors, such as oxidative stress (Vigna angularis [146]) or hypoxic stress (resveratrol [144]).

3.9. Herbal Products Apt to Activate Protective Pathways. The mechanisms able to delay the progression of DN include, 
besides autophagy, the pathways driven by PPARs [147], EP4/Gs (protein Gs)/AC/cAMP [148], Nrf2 [42, 58, 149], AMPK [71], adiponectin [150], and SIRT1 [74, 75].

3.9.1. In Vitro Studies-Potentially Nephroprotective Mechanisms. Activated by environmental stressors, p38MAPK stimulates cellular growth, differentiation, and apoptosis $[151,152]$. Activation of p38MAPK signaling is associated with the development of DN, being involved especially in the progression of interstitial fibrosis [11]. Emodin (from Rheum palmatum) interferes with p38MAPK pathway, thereby quelling proliferation and fibrosis, besides switching on the protective action of PPAR- $\gamma$ signaling system [153].

3.9.2. In Vivo Studies-Actually Nephroprotective Mechanisms. PPARs, important regulators of lipid and glucose metabolism, are ligand-activated nuclear transcription factors demonstrated to have a protective role in DN. PPAR- $\gamma$ ameliorates hyperglycemia, insulin resistance, hypertension, and albuminuria, and inactivates diacylglycerol (DAG)/protein kinase $\mathrm{C}(\mathrm{PKC}) /$ extracellular signal-regulated kinase (ERK) pathway with subsequent decrease in TGF- $\beta 1$, fibronectin, and type IV collagen. It also dampens inflammation and oxidative stress and blocks atheroma-promoting mechanisms. $P P A R-\alpha$ activation reduces TGF- $\beta 1$ and type IV collagen expression and prevents fatty acid build-up and lipidinduced toxicity in the diabetic kidneys [147].

Several herbal products are able to block the proliferation/fibrosis/sclerosis-inducing p38MAPK pathway, coupled with the activation of signaling pathways that may have protective effects, such as PPAR- $\gamma$ (Danhong, an extract of Salvia miltiorrhiza and Carthamus tinctorius [154]) or PI3K/Akt. The upregulation of the latter by (-)-epigallocatechin-3-gallate was accompanied by a favorable effect on the RAS and on the oxidative status [155]. However, Abelmoschus manihot extract decreased the level of p-Akt, while still exerting a renoprotective effect associated with quenching of the p38MAPK pathway and anti-inflammatory, antifibrosing, and antioxidant effects [156]. An experiment done on $d b / d b$ mice seems to indicate that Huangqi decoction shields the kidney from the consequences of diabetes through the agency of PI3K-Akt signaling. However, the activation of PI3K was associated with lower levels of the active form of Akt (p-Akt), which may explain why the positive effects of PI3K upregulation (higher expression of GLUT4 receptors that mediate intracellular glucose uptake) were not accompanied by the undesirable ones (protein synthesis and cellular proliferation driven by p-Akt) [36].

The flavonol myricetin activates $P P A R-\alpha$, improves the biological and histopathological markers of kidney injury and fibrosis, reduces the levels of SREBPs, and favorably interferes with lipid metabolism [157]. As mentioned above, the antifibrosis effect of Hypericum perforatum was accompanied by the activation of $P P A R-\gamma$ signaling pathway [31]. Abelmoschus manihot extract augmented the transcriptional activity of PPAR- $\alpha$ and PPAR- $\gamma$, improved the lipid status, and decreased the expression of inflammatory genes, ER stress, and c-Jun NH2-terminal kinase activation [104].
$E P 4 / G s / A C / c A M P$ signaling pathway (a Gs proteincoupled EP4 receptor that generates cAMP by activating AC) exerts a kidney-protective effect in diabetics ameliorating renal injury and slowing the progression of experimentally induced CKD [148]. At least some of the renoprotective effects of berberine are due to its ability to activate this pathway [158].

3.9.3. Human Studies-Actually Nephroprotective Mechanisms. A systematic review and meta-analysis that took into account 66 studies involving 4785 participants concluded that Astragalus membranaceus (Huang Qi) increased the effectiveness of conventional therapies in lowering albuminuria, proteinuria, and serum creatinine levels, with no increase in adverse effects. However, methodological flaws resulted in a low quality of the available studies [159].

\section{Strength of the Evidence in Various Areas}

The evidence provided by in vitro and in vivo animal studies is abundant and strong. Much fewer are the human studies. We have found 12 references regarding clinical trials on herbal products in DN. Only 5 of them included information about putative action mechanisms. Two of them are high quality (J5), one on silymarin (decrease in albuminuria) [65], the other on Tangshen Formula (decrease in proteinuria, increase in eGFR) $[124,125]$. The other three are low quality (J1): one on berberine (decrease in albuminuria) [95], one on Dioscorea bulbifera (decrease in proteinuria) [96], and one on Salacia chinensis (increased creatinine clearance, slowed CKD progression) [102]. Five other references describe study protocols on the efficiency of various traditional Chinese herbal medicines in DN [160-164]. The remaining two articles present the results of clinical trials that did not have as an objective the identification of action mechanisms. One is a moderate power randomized placebocontrolled trial demonstrating the ability of a Chinese herbal formula to decrease microalbuminuria [165]; the trial is of low-to-moderate quality, i.e., J2, as it was not doubleblinded (only the participants were blinded) and no information on dropouts/withdrawals was provided. The other one was a moderate-powered double-blinded, randomized, high-quality (J5) clinical trial on saffron (Crocus sativus L.) but failed to show any significant change in kidney function parameters or albuminuria [166].

A recent meta-analysis of randomized placebo-controlled trials of Chinese herbal medicine for DN selected 20 studies including 2719 patients and concluded that plant products may favorably influence renal function and albuminuria beyond the beneficial effect of conventional therapy (mainly RAS inhibitors). However, the evidence was deemed of moderate-to-low quality. The medicinal plants most frequently employed in the various combinations were Astragali radix, Rehmanniae Radix, and Rhei Radix et Rhizoma [167]. The most studied herbal in DN seems to be Astragalus membranaceus, but the available studies are of low quality [159].

We may conclude that most of the existing studies are of inferior quality, but there are a few moderate- or high-quality ones proving that herbals may help prevent kidney injury and maintain kidney function in diabetic patients. Some of 


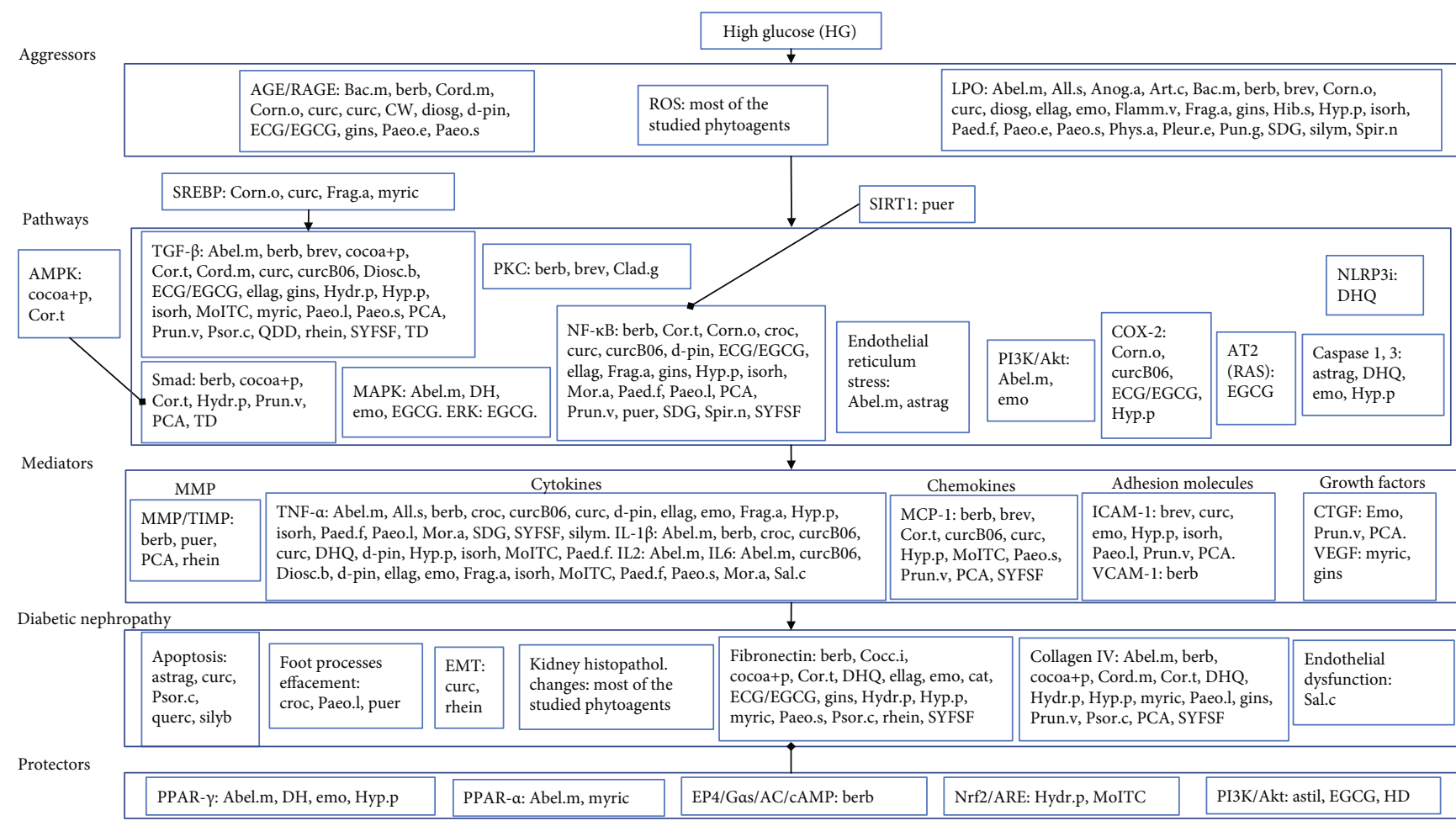

FIGURE 2: Graphical abstract: phytoagents active in diabetic nephropathy grouped according to their mechanism of action. Phytoagents: Abel.m: Abelmoschus manihot; All.s: Allium sativum; Anog.a: Anogeissus acuminata; Art.c: Artemisia campestris; astil: astilbin; astrag: astragaloside IV; Bac.m: Bacopa monnieri; berb: berberine; brev: breviscapine; cat: (+)-catechin; Chlor.p: Chlorella pyrenoidosa; Clad.g: Cladophora glomerata; Cocc.i: Coccinia indica; cocoa+p: cocoa enriched with polyphenols; Cor.t: Coreopsis tinctoria; Cord.m: Cordyceps militaris; Corn.o: Cornus officinalis; croc: crocin; curc: curcumin; curcB06: curcumin analogue B06; CW: coconut water; DH: Danhong; DHQ: dihydroquercetin; Diosc.b: Dioscorea bulbifera; diosg: diosgenin; d-pin: d-pinitol; ECG: (-)-epicatechin 3-O-gallate (Camellia sinensis); EGCG: (-)-epigallocatechin 3-O-gallate; ellag: ellagic acid; emo: emodin; Flamm.v: Flammulina velutipes; Frag.a: Fragaria $\times$ ananassa; gins: ginseng; HD: Huangqi decoction; Hib.s: Hibiscus sabdariffa; Hydr.p: Hydrangea paniculata; Hyp.p: Hypericum perforatum; isorh: isorhamnetin; MoITC: moringa isothiocyanate; Mor.a: Morus alba; myric: myricetin; Paed.f: Paederia foetida; Paeo.e: Paeonia emodi; Paeo.l: Paeonia lactiflora; Paeo.s: Paeonia suffruticosa; PCA: purple corn anthocyanins; Phys.a: Physalis angulata; Pleur.e: Pleurotus eryngii; Prun.v: Prunella vulgaris; Psor.c: Psoralea corylifolia; puer: puerarin; Pun.g: Punica granatum; QDD: Qidan Dihuang decoction; querc: quercetin; Rhe.r: Rheum ribes; Sal.c: Salacia chinensis; SDG: secoisolariciresinol diglucoside; silyb: silybin; silym: silymarin; Spir.n: Spirogyra neglecta; SYFSF: Shen-Yan-Fang-Shuai Formula; TD: Tangke decoction.

the most promising phytoceuticals are silymarin, Astragalus membranaceus, and Tangshen Formula. Given the vast experience with silymarin in the treatment of other (mainly liver) disorders, the wide availability of financially affordable silymarin containing preparations, and the lack of side effects, at least silymarin should be probably included in the standard treatment of diabetic patients, pending the completion of ongoing clinical trials on various other herbal combinations.

\section{Concluding Remarks}

Many agents of plant origin have shown the ability to prevent $\mathrm{DN}$, the mechanisms involved being, to a great extent, similar to those of the conventional drugs used for this aim: blocking RAAS, antioxidant, anti-inflammatory, and antiproliferative, preventing sclerosis/fibrosis, improving lipid profile, and activating protective pathways. Consequently, herbal medicines may emerge as healthy alternatives to the agents cur-

rently employed for slowing the progression of DN. The most important setback is the scarcity of human experiments, which is surprising and regrettable as these agents are generally devoid of side effects. The dearth of human studies is compounded by the low quality of most of the existing ones. Both these issues should be urgently addressed by future research in this area. A graphical abstract of this paper is provided as Figure 2.

\section{Abbreviations}

AC:

AGEs:

AMPK:

cAMP:

COX-2:

$\mathrm{DN}$ :

ECM:
Adenylyl/adenylate cyclase

Advanced glycation end-products

$5^{\prime}$ adenosine monophosphate-activated protein kinase

Cyclic adenosine monophosphate

Cyclooxygenase 2

Diabetic nephropathy

Extracellular matrix 


\begin{tabular}{|c|c|}
\hline eGFR: & Estimated glomerular filtration rate \\
\hline EMT: & $\begin{array}{l}\text { Epithelial-mesenchymal } \\
\text { transdifferentiation }\end{array}$ \\
\hline EP4: & E prostanoid receptor 4 \\
\hline $\mathrm{G} \alpha \mathrm{s}:$ & $\mathrm{G} \alpha$ s protein \\
\hline ERK: & Extracellular signal-regulated kinase \\
\hline ER: & Endoplasmic reticulum \\
\hline Gs: & Gs protein \\
\hline GSK: & Glycogen synthase kinase \\
\hline HG: & High glucose \\
\hline ICAM: & Intercellular adhesion molecule \\
\hline IL: & Interleukin \\
\hline ILK: & Integrin-linked kinase \\
\hline $\mathrm{J} 1 / \mathrm{J} 2 / \mathrm{J} 3 / \mathrm{J} 4 / \mathrm{J} 5:$ & $\begin{array}{l}\text { Score of } 1 / 2 / 3 / 4 / 5 \text { on the Jadad scoring } \\
\text { system for the assessment of clinical trial } \\
\text { quality }\end{array}$ \\
\hline JNK: & c-Jun N-terminal kinase \\
\hline LPO: & Lipid peroxidation \\
\hline MAPK: & Mitogen-activated protein kinase \\
\hline MCP: & $\begin{array}{l}\text { Monocyte chemoattractant/chemotactic } \\
\text { protein }\end{array}$ \\
\hline MMP: & Matrix metalloproteinase \\
\hline mTOR: & $\begin{array}{l}\text { Mammalian/mechanistic target of } \\
\text { rapamycin }\end{array}$ \\
\hline NADPH: & $\begin{array}{l}\text { Reduced nicotinamide-adenine } \\
\text { dinucleotide phosphate }\end{array}$ \\
\hline $\mathrm{NF}-\kappa \mathrm{B}:$ & $\begin{array}{l}\text { Nuclear factor kappa-B (nuclear factor } \\
\text { kappa-light-chain-enhancer of activated B } \\
\text { cells) }\end{array}$ \\
\hline NO: & Nitric oxide \\
\hline NOX4: & NADPH oxidase 4 \\
\hline Nrf2: & Nuclear factor erythroid-derived 2 \\
\hline p-: & Phospho-/phosphorylated \\
\hline PI3K: & Phosphatidylinositol 3-kinase \\
\hline PKC: & Proteinkinase C \\
\hline PPAR: & Peroxisome proliferator-activated receptor \\
\hline RAGE: & Receptor for AGEs \\
\hline RAS: & Renin-angiotensin system \\
\hline ROS: & Reactive oxygen species \\
\hline SIRT1: & Sirtuin 1 \\
\hline Smad proteins: & $\begin{array}{l}\text { Signal transducers for receptors of TGF- } \beta \\
\text { superfamily, critically important for } \\
\text { regulating cell development and growth }\end{array}$ \\
\hline SREBP: & Sterol regulatory element-binding protein \\
\hline TGF- $\beta 1:$ & Transforming/tumor growth factor- $\beta 1$ \\
\hline TIMP: & Tissue inhibitor of MMPs \\
\hline TNF- $\alpha$ : & Tumor necrosis factor- $\alpha$. \\
\hline
\end{tabular}

\section{Conflicts of Interest}

The authors have declared that no conflicts of interest exist.

\section{Authors' Contributions}

Dorin Dragoș, Maria Mirabela Manea, Delia Timofte, and Dorin Ionescu have equally contributed to the conception of this paper and should be considered first authors.

\section{References}

[1] R. Z. Alicic, M. T. Rooney, and K. R. Tuttle, "Diabetic kidney disease: challenges, progress, and possibilities," Clinical Journal of the American Society of Nephrology, vol. 12, no. 12, pp. 2032-2045, 2017.

[2] S. Sivakumar, P. Palsamy, and S. P. Subramanian, "Impact of d-pinitol on the attenuation of proinflammatory cytokines, hyperglycemia-mediated oxidative stress and protection of kidney tissue ultrastructure in streptozotocin-induced diabetic rats," Chemico-Biological Interactions, vol. 188, no. 1, pp. 237-245, 2010.

[3] M. Hase, T. Babazono, S. Karibe, N. Kinae, and Y. Iwamoto, "Renoprotective effects of tea catechin in streptozotocininduced diabetic rats," International Urology and Nephrology, vol. 38, no. 3-4, pp. 693-699, 2007.

[4] C. Yan, W. Xu, Y. Huang et al., "HRD1-mediated IGF-1R ubiquitination contributes to renal protection of resveratrol in db/db mice," Molecular Endocrinology, vol. 30, no. 6, pp. 600-613, 2016.

[5] S. Ohga, K. Shikata, K. Yozai et al., "Thiazolidinedione ameliorates renal injury in experimental diabetic rats through anti-inflammatory effects mediated by inhibition of NF- $\kappa \mathrm{B}$ activation," American Journal of Physiology-Renal Physiolog, vol. 292, no. 4, pp. F1141-F1150, 2007.

[6] N. Banba, T. Nakamura, M. Matsumura, H. Kuroda, Y. Hattori, and K. Kasai, "Possible relationship of monocyte chemoattractant protein-1 with diabetic nephropathy," Kidney International, vol. 58, no. 2, pp. 684-690, 2000.

[7] Y. Qian, E. Feldman, S. Pennathur, M. Kretzler, and F. C. Brosius, "From fibrosis to sclerosis: mechanisms of glomerulosclerosis in diabetic nephropathy," Diabetes, vol. 57, no. 6, pp. 1439-1445, 2008.

[8] R. M. Mason and N. A. Wahab, "Extracellular matrix metabolism in diabetic nephropathy," Journal of the American Society of Nephrology, vol. 14, no. 5, pp. 1358-1373, 2003.

[9] K. O. Alsaad and A. M. Herzenberg, "Distinguishing diabetic nephropathy from other causes of glomerulosclerosis: an update," Journal of Clinical Pathology, vol. 60, no. 1, pp. 1826, 2007.

[10] S. Chen, B. Jim, and F. N. Ziyadeh, "Diabetic nephropathy and transforming growth factor- $\beta$ : transforming our view of glomerulosclerosis and fibrosis build-up," Seminars in Nephrology, vol. 23, no. 6, pp. 532-543, 2003.

[11] L. Adhikary, F. Chow, D. J. Nikolic-Paterson et al., "Abnormal p38 mitogen-activated protein kinase signalling in human and experimental diabetic nephropathy," Diabetologia, vol. 47, no. 7, pp. 1210-1222, 2004.

[12] A. Lan and J. Du, "Potential role of Akt signaling in chronic kidney disease," Nephrology Dialysis Transplantation, vol. 30, no. 3, pp. 385-394, 2015.

[13] A. Maffei, G. Lembo, and D. Carnevale, "PI3Kinases in diabetes mellitus and its related complications," International Journal of Molecular Sciences, vol. 19, no. 12, p. 4098, 2018.

[14] A. Lerman and A. M. Zeiher, "Endothelial function," Circulation, vol. 111, no. 3, pp. 363-368, 2005.

[15] J. H. Lin, P. Walter, and T. S. B. Yen, "Endoplasmic reticulum stress in disease pathogenesis," Annual Review of Pathology: Mechanisms of Disease, vol. 3, no. 1, pp. 399-425, 2008.

[16] Z. S. Wang, F. Xiong, X. H. Xie, D. Chen, J. H. Pan, and L. Cheng, "Astragaloside IV attenuates proteinuria in 
streptozotocin-induced diabetic nephropathy via the inhibition of endoplasmic reticulum stress," BMC Nephrology, vol. 16, no. 1, 2015.

[17] M. E. Molitch, A. I. Adler, A. Flyvbjerg et al., "Diabetic kidney disease: a clinical update from kidney disease: improving global outcomes," Kidney International, vol. 87, no. 1, pp. 20-30, 2015.

[18] L.-J. Sun, Y.-N. Sun, J.-P. Shan, and G.-R. Jiang, "Effects of mineralocorticoid receptor antagonists on the progression of diabetic nephropathy," Journal of Diabetes Investigation, vol. 8, no. 4, pp. 609-618, 2017.

[19] K. Shapiro and W. C. Gong, "Natural products used for diabetes," Journal of the American Pharmaceutical Association (1996), vol. 42, no. 2, pp. 217-226, 2002.

[20] A. Gordon, Z. Buch, V. Baute, and R. Coeytaux, "Use of ayurveda in the treatment of type 2 diabetes mellitus," Global Advances in Health and Medicine, vol. 8, article $216495611986109,2019$.

[21] J. Tian, F. Lian, X. Yu et al., "The efficacy and safety of Chinese herbal decoction in type 2 diabetes: a 5-year retrospective study," Evidence Based Complement and Alternative Medicine, vol. 2016, article 5473015, 8 pages, 2016.

[22] S. Telapolu, M. Kalachavedu, A. M. Punnoose, and D. Bilikere, "MD-1, a poly herbal formulation indicated in diabetes mellitus ameliorates glucose uptake and inhibits adipogenesis - an in vitro study," BMC Complementary and Alternative Medicine, vol. 18, no. 1, p. 113, 2018.

[23] C. L. T. Chang, Y. Lin, A. P. Bartolome, Y.-C. Chen, S.C. Chiu, and W.-C. Yang, "Herbal therapies for type 2 diabetes mellitus: chemistry, biology, and potential application of selected plants and compounds," Evidence Based Complementary and Alternative Medicine, vol. 2013, article 378657, 33 pages, 2013.

[24] A. B. Medagama, R. Bandara, R. A. Abeysekera, B. Imbulpitiya, and T. Pushpakumari, "Use of complementary and alternative medicines (CAMs) among type 2 diabetes patients in Sri Lanka: a cross sectional survey," BMC Complementary and Alternative Medicine, vol. 14, no. 1, 2014.

[25] V. W. Berger and S. Y. Alperson, "A general framework for the evaluation of clinical trial quality," Reviews on Recent Clinical Trials, vol. 4, no. 2, pp. 79-88, 2009.

[26] T. S. Park, "How much glycemic control is needed to prevent progression of diabetic nephropathy?," Journa of Diabetes Investigation, vol. 3, no. 5, pp. 411-412, 2012.

[27] A. Whaley-Connell and J. R. Sowers, "Insulin resistance in kidney disease: is there a distinct role separate from that of diabetes or obesity?," Cardiorenal Medicine, vol. 8, no. 1, pp. 41-49, 2017.

[28] N. Ziamajidi, A. Nasiri, R. Abbasalipourkabir, and S. Sadeghi Moheb, "Effects of garlic extract on TNF- $\alpha$ expression and oxidative stress status in the kidneys of rats with STZ + nicotinamide-induced diabetes," Pharmaceutical Biology, vol. 55, no. 1, pp. 526-531, 2016.

[29] F. Irshaid, K. Mansi, A. Bani-Khaled, and T. Aburjia, "Hepatoprotetive, Cardioprotective and nephroprotective actions of essential oil extract of Artemisia sieberi in alloxan induced diabetic rats," Iranian Journal of Pharmaceutical Research, vol. 11, no. 4, pp. 1227-1234, 2012.

[30] L. Kishore, N. Kaur, and R. Singh, "Renoprotective effect of Bacopa monnieri via inhibition of advanced glycation end products and oxidative stress in STZ-nicotinamide-induced diabetic nephropathy," Renal Failure, vol. 38, no. 9, pp. 1528-1544, 2016.

[31] D. M. Abd El Motteleb and D. I. Abd El Aleem, "Renoprotective effect of Hypericum perforatum against diabetic nephropathy in rats: insights in the underlying mechanisms," Clinical and Experimental Pharmacology and Physiology, vol. 44, no. 4, pp. 509-521, 2017.

[32] P. Ankita, B. Deepti, and M. Nilam, "Flavonoid rich fraction of Punica granatum improves early diabetic nephropathy by ameliorating proteinuria and disturbed glucose homeostasis in experimental animals," Pharmaceutical Biology, vol. 53, no. 1, pp. 61-71, 2014.

[33] N. K. Rao and S. Nammi, "Antidiabetic and renoprotective effects of the chloroform extract of Terminalia chebula Retz. seeds in streptozotocin-induced diabetic rats," BMC Complementary and Alternative Medicine, vol. 6, no. 1, 2006.

[34] C. Srimaroeng, A. Ontawong, N. Saowakon et al., "Antidiabetic and renoprotective effects of Cladophora glomerata Kützing extract in experimental type 2 diabetic rats: a potential nutraceutical product for diabetic nephropathy," Journal of Diabetes Research, vol. 2015, Article ID 320167, 15 pages, 2015.

[35] Z.-H. Chen, J. Li, J. Liu et al., "Saponins isolated from the root ofPanax notoginsengShowed significant anti-diabetic effects in KK-Ay mice," The American Journal of Chinese Medicine, vol. 36, no. 5, pp. 939-951, 2012.

[36] X. Chen, H. Wang, M. Jiang et al., "Huangqi (astragalus) decoction ameliorates diabetic nephropathy via IRS1-PI3KGLUT signaling pathway," American Journal of Translational Research, vol. 10, no. 8, pp. 2491-2501, 2018.

[37] A. Ahad, A. A. Ganai, M. Mujeeb, and W. A. Siddiqui, "Ellagic acid, an NF- $\kappa \mathrm{B}$ inhibitor, ameliorates renal function in experimental diabetic nephropathy," Chemico-Biological Interactions, vol. 219, pp. 64-75, 2014.

[38] H. Ha, I.-A. Hwang, J. H. Park, and H. B. Lee, "Role of reactive oxygen species in the pathogenesis of diabetic nephropathy," Diabetes Research and Clinical Practice, vol. 82, pp. S42S45, 2008.

[39] C. W. Yang, H. Vlassara, E. P. Peten, C. J. He, G. E. Striker, and L. J. Striker, "Advanced glycation end products upregulate gene expression found in diabetic glomerular disease," Proceedings of the National Academy of Sciences, vol. 91, no. 20, pp. 9436-9440, 1994.

[40] M. Zhang, L. Feng, M. Zhu, J. Gu, C. Wu, and X. Jia, “Antioxidative and anti-inflammatory activities of paeoniflorin and oxypaeoniflora on AGEs-induced mesangial cell damage," Planta Medica, vol. 79, no. 14, pp. 1319-1323, 2013.

[41] J. Liu, L. Feng, M. Zhu et al., "The in vitro protective effects of curcumin and demethoxycurcumin in curcuma longa extract on advanced glycation end products-induced mesangial cell apoptosis and oxidative stress," Planta Medica, vol. 78, no. 16, pp. 1757-1760, 2012.

[42] D. Cheng, L. Gao, S. Su et al., "Moringa isothiocyanate activates Nrf2: potential role in diabetic nephropathy," The AAPS Journal, vol. 21, no. 2, p. 31, 2019.

[43] K. Khazim, Y. Gorin, R. C. Cavaglieri, H. E. Abboud, and P. Fanti, "The antioxidant silybin prevents high glucoseinduced oxidative stress and podocyte injury in vitro and in vivo," American Journal of Physiology-Renal Physiology, vol. 305, no. 5, pp. F691-F700, 2013. 
[44] X. Zhang, D. Liang, X. Lian et al., "Berberine activates Nrf2 nuclear translocation and inhibits apoptosis induced by high glucose in renal tubular epithelial cells through a phosphatidylinositol 3-kinase/Akt-dependent mechanism," Apoptosis, vol. 21, no. 6, pp. 721-736, 2016.

[45] X. Zhang, T. Guan, B. Yang, Z. Chi, Q. Wan, and H. F. Gu, "Protective effect of berberine on high glucose and hypoxiainduced apoptosis via the modulation of HIF- $1 \alpha$ in renal tubular epithelial cells," American Journal of Translational Research, vol. 11, no. 2, pp. 669-682, 2019.

[46] S. P. Chennasamudram, S. Kudugunti, P. R. Boreddy, M. Y. Moridani, and T. L. Vasylyeva, "Renoprotective effects of (+)-catechin in streptozotocin-induced diabetic rat model," Nutrition Research, vol. 32, no. 5, pp. 347-356, 2012.

[47] T. Yokozawa, K. S. Kang, C. H. Park et al., "Bioactive constituents of Corni Fructus: the therapeutic use of morroniside, loganin, and 7-O-galloyl-D-sedoheptulose as renoprotective agents in type 2 diabetes," Drug Discoverie \& Therapeutic, vol. 4, no. 4, pp. 223-234, 2010.

[48] T. Ding, S. Wang, X. Zhang et al., "Kidney protection effects of dihydroquercetin on diabetic nephropathy through suppressing ROS and NLRP3 inflammasome," Phytomedicine, vol. 41, pp. 45-53, 2018.

[49] I. B. S. Gomes, M. L. Porto, M. C. L. F. S. Santos et al., "Renoprotective, anti-oxidative and anti-apoptotic effects of oral low-dose quercetin in the C57BL/6J model of diabetic nephropathy," Lipids Health and Disease, vol. 13, no. 1, p. 184, 2014.

[50] S. Siddiqui, M. Rashid Khan, and W. A. Siddiqui, "Comparative hypoglycemic and nephroprotective effects of tocotrienol rich fraction (TRF) from palm oil and rice bran oil against hyperglycemia induced nephropathy in type 1 diabetic rats," Chemico-Biological Interactions, vol. 188, no. 3, pp. 651-658, 2010.

[51] X. M. Qi, G. Z. Wu, Y. G. Wu, H. Lin, J. J. Shen, and S. Y. Lin, "Renoprotective effect of breviscapine through suppression of renal macrophage recruitment in streptozotocin-induced diabetic rats," Nephron Experimental Nephrology, vol. 104, no. 4, pp. e147-e157, 2006.

[52] X.-X. Xu, W. Zhang, P. Zhang, X.-M. Qi, Y.-G. Wu, and J.J. Shen, "Superior renoprotective effects of the combination of breviscapine with enalapril and its mechanism in diabetic rats," Phytomedicine, vol. 20, no. 10, pp. 820-827, 2013.

[53] L. Lin, F. Cui, J. Zhang et al., "Antioxidative and renoprotective effects of residue polysaccharides from Flammulina velutipes," Carbohydrate Polymers, vol. 146, pp. 388-395, 2016.

[54] Y. Seujange, A. Leelahavanichkul, W. Yisarakun et al., "Hibiscus SabdariffaLinnaeus aqueous extracts attenuate the progression of renal injury in 5/6 nephrectomy rats," Renal Failure, vol. 35, no. 1, pp. 118-125, 2012.

[55] M. Sefi, H. Fetoui, N. Soudani, Y. Chtourou, M. Makni, and N. Zeghal, "Artemisia campestris leaf extract alleviates early diabetic nephropathy in rats by inhibiting protein oxidation and nitric oxide end products," Pathology - Research and Practice, vol. 208, no. 3, pp. 157-162, 2012.

[56] A. M. Navale and A. Paranjape, "Antidiabetic and renoprotective effect of Anogeissus acuminata leaf extract on experimentally induced diabetic nephropathy," Journal of Basic and Clinical Physiology and Pharmacology, vol. 29, no. 4, pp. 359364, 2018.

[57] C. Zhang, L. Zhang, H. Liu, J. Zhang, C. Hu, and L. Jia, “Antioxidation, anti-hyperglycaemia and renoprotective effects of extracellular polysaccharides from Pleurotus eryngii SI-04," International Journal of Biological Macromolecules, vol. 111, pp. 219-228, 2018.

[58] J. Trujillo, Y. I. Chirino, E. Molina-Jijón, A. C. AndéricaRomero, E. Tapia, and J. Pedraza-Chaverrí, "Renoprotective effect of the antioxidant curcumin: recent findings," Redox Biology, vol. 1, no. 1, pp. 448-456, 2013.

[59] Z. Sen, W. Weida, M. Jie, S. Li, Z. Dongming, and C. Xiaoguang, "Coumarin glycosides from Hydrangea paniculata slow down the progression of diabetic nephropathy by targeting Nrf2 anti-oxidation and smad2/3-mediated profibrosis," Phytomedicine, vol. 57, pp. 385-395, 2019.

[60] Y. Zhong, X. Zhang, X. Cai, K. Wang, Y. Chen, and Y. Deng, "Puerarin attenuated early diabetic kidney injury through down-regulation of matrix metalloproteinase 9 in streptozotocin-induced diabetic rats," PLoS One, vol. 9, no. 1, article e85690, 2014.

[61] I. F. D. Pinto, R. P. Silva, A. d. B. C. Filho et al., "Study of Antiglycation, Hypoglycemic, and Nephroprotective Activities of the Green Dwarf Variety Coconut Water (Cocos nucifera L.) in Alloxan-Induced Diabetic Rats," Journal of Medicinal Food, vol. 18, no. 7, pp. 802-809, 2015.

[62] D. M. Kanchan, G. S. Somani, V. V. Peshattiwar, A. A. Kaikini, and S. Sathaye, "Renoprotective effect of diosgenin in streptozotocin induced diabetic rats," Pharmacological Reports, vol. 68, no. 2, pp. 370-377, 2016.

[63] L. Kishore, N. Kaur, and R. Singh, "Nephroprotective effect of Paeonia emodi via inhibition of advanced glycation end products and oxidative stress in streptozotocin -nicotinamide induced diabetic nephropathy," Journal of Food and Drug Analysis, vol. 25, no. 3, pp. 576-588, 2017.

[64] E. O. Adewoye, M. A. Oguntola, and A. O. Ige, "Anti-oxidative and reno-restorative effects of physalis angulata (whole plant extract) in alloxan-induced diabetic male Wistar rats," African Journal of Medicine and Medical Sciences, vol. 45, no. 1, pp. 99-108, 2016.

[65] M. K. Fallahzadeh, B. Dormanesh, M. M. Sagheb et al., "Effect of Addition of Silymarin to Renin-Angiotensin System Inhibitors on Proteinuria in Type 2 Diabetic Patients With Overt Nephropathy: A Randomized, Double-Blind, PlaceboControlled Trial," American Journal of Kidney Diseases, vol. 60, no. 6, pp. 896-903, 2012.

[66] Y. Pan, G. Zhu, Y. Wang et al., "Attenuation of high-glucoseinduced inflammatory response by a novel curcumin derivative B06 contributes to its protection from diabetic pathogenic changes in rat kidney and heart," The Journal of Nutritional BiochemistryThe Journal of Nutritional Biochemistry, vol. 24, no. 1, pp. 146-155, 2013.

[67] T. Fujita, S. Hemmi, M. Kajiwara et al., "Complement-mediated chronic inflammation is associated with diabetic microvascular complication," Diabetes/Metabolism Research and Reviews, vol. 29, no. 3, pp. 220-226, 2013.

[68] M. B. Duran-Salgado and A. F. Rubio-Guerra, "Diabetic nephropathy and inflammation," World Journal of Diabetes, vol. 5, no. 3, pp. 393-398, 2014.

[69] J. Guo, Y. Gao, Y. Wang, W. J. Liu, J. Zhou, and Z. Wang, "Application of Herbal Medicines with Heat-Clearing Property to Anti- Microinflammation in the Treatment of Diabetic Kidney Disease," Evidence-Based Complementary and Alternative Medicine, vol. 2019, Article ID 6174350, 6 pages, 2019.

[70] J. Li, S. S. Lim, J.-Y. Lee et al., "Purple corn anthocyanins dampened high-glucose-induced mesangial fibrosis and 
inflammation: possible renoprotective role in diabetic nephropathy," The Journal of Nutritional Biochemistry, vol. 23, no. 4, pp. 320-331, 2012.

[71] J. Zhao, Ed.et al., "AMP-activated protein kinase (AMPK) activation inhibits nuclear translocation of Smad4 in mesangial cells and diabetic kidneys," American Journal of Physiology-Renal Physiology, vol. 308, no. 10, pp. F1167F1177, 2015.

[72] L. Yao, J. Li, L. Li et al., "Coreopsis tinctoria Nutt ameliorates high glucose-induced renal fibrosis and inflammation via the TGF- $\beta 1 /$ SMADS/AMPK/NF- $\kappa$ B pathways," BMC Complementary and Alternative Medicine, vol. 19, no. 1, p. 14, 2019.

[73] S. Li, X. Liu, J. Lei, J. Yang, P. Tian, and Y. Gao, "Crocin Protects Podocytes Against Oxidative Stress and Inflammation Induced by High Glucose Through Inhibition of NF- $\kappa \mathrm{B}$," Cellular Physiology and Biochemistry, vol. 42, no. 4, pp. 14811492, 2017.

[74] F. Yeung, J. E. Hoberg, C. S. Ramsey et al., "Modulation of NF- $\kappa$ B-dependent transcription and cell survival by the SIRT1 deacetylase," The EMBO Journal, vol. 23, no. 12, pp. 2369-2380, 2004.

[75] Y. Zhong, K. Lee, and J. C. He, "SIRT1 Is a Potential Drug Target for Treatment of Diabetic Kidney Disease," Frontiers in Endocrinology, vol. 9, 2018.

[76] X. Li, W. Cai, K. Lee et al., "Publisher Correction: Puerarin attenuates diabetic kidney injury through the suppression of NOX4 expression in podocytes," Scientific Reports, vol. 7, no. 1, article 18113, 2017.

[77] C. Guo, T. Liang, Q. He, P. Wei, N. Zheng, and L. Xu, "Renoprotective effect of ramulus mori polysaccharides on renal injury in STZ- diabetic mice," International Journal of Biological Macromolecules, vol. 62, pp. 720-725, 2013.

[78] M. P. Borgohain, L. Chowdhury, S. Ahmed et al., "Renoprotective and antioxidative effects of methanolic Paederia foetida leaf extract on experimental diabetic nephropathy in rats," Journal of Ethnopharmacology, vol. 198, pp. 451-459, 2017.

[79] V. Soetikno, F. R. Sari, P. T. Veeraveedu et al., "Curcumin ameliorates macrophage infiltration by inhibiting NF- $\kappa \mathrm{B}$ activation and proinflammatory cytokines in streptozotocin induced-diabetic nephropathy," Nutrition \& Metabolism, vol. 8, no. 1, p. 35, 2011.

[80] J. Lv, Z. Wang, Y. Wang et al., "Renoprotective Effect of the Shen-Yan-Fang-Shuai Formula by Inhibiting TNF- $\alpha / \mathrm{NF}-\kappa \mathrm{B}$ Signaling Pathway in Diabetic Rats," Journal of Diabetes Research, vol. 2017, Article ID 4319057, 11 pages, 2017.

[81] T. Yokozawa, J. S. Noh, and C. H. Park, "Green Tea Polyphenols for the Protection against Renal Damage Caused by Oxidative Stress," Evidence-Based Complementary and Alternative Medicine, vol. 2012, Article ID 845917, 12 pages, 2012.

[82] S. Sen, S. Chen, B. Feng, Y. Wu, E. Lui, and S. Chakrabarti, "Preventive effects of North American ginseng (Panax quinquefolium) on diabetic nephropathy," Phytomedicine, vol. 19, no. 6, pp. 494-505, 2012.

[83] H. Y. Kim, K. S. Kang, N. Yamabe, R. Nagai, and T. Yokozawa, "Protective Effect of Heat-Processed American Ginseng against Diabetic Renal Damage in Rats," Journal of Agricultural and Food Chemistry, vol. 55, no. 21, pp. 84918497, 2007.

[84] S. Namgung, J. J. Yoon, C.-S. Yoon et al., "Prunella vulgarisAttenuates Diabetic Renal Injury by Suppressing Glomeru- lar Fibrosis and Inflammation," The American Journal of Chinese Medicine, vol. 45, no. 3, pp. 475-495, 2017.

[85] S.-F. Sun, T.-T. Zhao, H.-J. Zhang et al., "Renoprotective effect of berberine on type 2 diabetic nephropathy in rats," Clinical and Experimental Pharmacology and Physiology, vol. 42, no. 6, pp. 662-670, 2015.

[86] Y. Zhong, K. Lee, Y. Deng et al., "Arctigenin attenuates diabetic kidney disease through the activation of PP2A in podocytes," Nature Communications, vol. 10, no. 1, p. 4523, 2019.

[87] S. Qiu, G. Sun, Y. Zhang, X. Li, and R. Wang, "Involvement of the NF- $\kappa$ B signaling pathway in the renoprotective effects of isorhamnetin in a type 2 diabetic rat model," Biomedical Reports, vol. 4, no. 5, pp. 628-634, 2016.

[88] I. O. Sherif, "Secoisolariciresinol diglucoside in high-fat diet and streptozotocin-induced diabetic nephropathy in rats: a possible renoprotective effect," Journal of Physiology and Biochemistry, vol. 70, no. 4, pp. 961-969, 2014.

[89] P. Mandave, S. Khadke, M. Karandikar et al., "Antidiabetic, Lipid Normalizing, and Nephroprotective Actions of the Strawberry: A Potent Supplementary Fruit," International Journal of Molecular Sciences, vol. 18, no. 1, p. 124, 2017.

[90] L. Uttarwar, B. Gao, A. J. Ingram, and J. C. Krepinsky, "SREBP-1 activation by glucose mediates TGF- $\beta$ upregulation in mesangial cells," American Journal of PhysiologyRenal Physiology, vol. 302, no. 3, pp. F329-F341, 2012.

[91] Y. Yang, H. Wang, M. Kouadir, H. Song, and F. Shi, "Recent advances in the mechanisms of NLRP3 inflammasome activation and its inhibitors," Cell Death \& Disease, vol. 10, no. 2, p. 128, 2019.

[92] M. M. Mariappan, S. Prasad, K. D'Silva et al., "Activation of glycogen synthase Kinase $3 \beta$ ameliorates diabetes-induced kidney injury," Journal of Biological Chemistry, vol. 289, no. 51, pp. 35363-35375, 2014.

[93] B. A. Hemmings and D. F. Restuccia, "PI3K-PKB/Akt Pathway," Cold Spring Harbor Perspectives in Biology, vol. 4, no. 9, 2012.

[94] D. Jing, H. Bai, and S. Yin, "Renoprotective effects of emodin against diabetic nephropathy in rat models are mediated via PI3K/Akt/GSK-3 $\beta$ and Bax/caspase-3 signaling pathways," Experimental and Therapeutic Medicine, vol. 14, no. 5, pp. 5163-5169, 2017.

[95] P. Dai, J. Wang, L. Lin, Y. Zhang, and Z. Wang, "Renoprotective effects of berberine as adjuvant therapy for hypertensive patients with type 2 diabetes mellitus: Evaluation via biochemical markers and color Doppler ultrasonography," Experimental and Therapeutic Medicine, vol. 10, no. 3, pp. 869-876, 2015.

[96] R. G. Singh, M. Rajak, B. Ghosh, A. A. Usha, and G. P. Dubey, "Comparative evaluation of fosinopril and herbal drug Dioscorea bulbifera in patients of diabetic nephropathy," Saudi Journal of Kidney Diseases and Transplantation, vol. 24, no. 4, pp. 737-742, 2013.

[97] P. Paulus, C. Jennewein, and K. Zacharowski, "Biomarkers of endothelial dysfunction: can they help us deciphering systemic inflammation and sepsis?," Biomarkers, vol. 16, pp. S11-S21, 2011.

[98] S. Pushpakumar, S. Kundu, and U. Sen, "Endothelial Dysfunction: The Link Between Homocysteine and Hydrogen Sulfide," Current Medicinal Chemistry, vol. 21, no. 32, pp. 3662-3672, 2014. 
[99] E. Esteve, A. Castro, A. Lopez-Bermejo, J. Vendrell, W. Ricart, and J. M. Fernandez-Real, "Serum Interleukin-6 Correlates With Endothelial Dysfunction in Healthy Men Independently of Insulin Sensitivity," Diabetes Care, vol. 30, no. 4, pp. 939-945, 2007.

[100] P. Moreau, L. V. d'Uscio, S. Shaw, H. Takase, M. Barton, and T. F. Lüscher, "Angiotensin II Increases Tissue Endothelin and Induces Vascular Hypertrophy," Circulation, vol. 96, no. 5, pp. 1593-1597, 1997.

[101] T. E. Hunley and V. Kon, "Glomerular Filtration: Still Sympathetic to Endothelin's Influence?," Journal of the American Society of Nephrology, vol. 20, no. 7, pp. 1427-1429, 2009.

[102] S. S. Rathore, Usha, G. P. Dubey, R. G. Singh, R. Kumar, and A. Agarwal, "Nephroprotective role of salacia chinensis in diabetic ckd patients: A pilot study," Indian Journal of Medical Sciences, vol. 64, no. 8, p. 378, 2010.

[103] H. Guo, Y. Wang, X. Zhang et al., "Astragaloside IV protects against podocyte injury via SERCA2-dependent ER stress reduction and AMPK $\alpha$-regulated autophagy induction in streptozotocin- induced diabetic nephropathy," Scientific Reports, vol. 7, no. 1, p. 6852, 2017.

[104] J. Ge, J.-J. Miao, X.-Y. Sun, and J.-Y. Yu, "Huangkui capsule, an extract from Abelmoschus manihot (L.) medic, improves diabetic nephropathy via activating peroxisome proliferator -activated receptor (PPAR)- $\alpha / \gamma$ and attenuating endoplasmic reticulum stress in rats," Journal of Ethnopharmacology, vol. 189, pp. 238-249, 2016.

[105] D. P. Basile, "Transforming growth factor- $\beta$ as a target for treatment in diabetic nephropathy," American Journal of Kidney Diseases, vol. 38, no. 4, pp. 887-890, 2001.

[106] M. K. Wendt, T. M. Allington, and W. P. Schiemann, "Mechanisms of the epithelial-mesenchymal transition by TGF- $\beta$," Future Oncology, vol. 5, no. 8, pp. 1145-1168, 2009.

[107] K. Sharma and F. N. Ziyadeh, "Hyperglycemia and Diabetic Kidney Disease: The Case for Transforming Growth Factoras a Key Mediator," Diabetes, vol. 44, no. 10, pp. 11391146, 1995.

[108] Z. S. Ibrahim, M. E. Alkafafy, M. M. Ahmed, and M. M. Soliman, "Renoprotective effect of curcumin against the combined oxidative stress of diabetes and nicotine in rats," Molecular Medicine Reports, vol. 13, no. 4, pp. 3017-3026, 2016.

[109] M. Sun, L. Huang, J. Zhu, W. Bu, J. Sun, and Z. Fang, "Screening nephroprotective compounds from cortex Moutan by mesangial cell extraction and UPLC," Archives of Pharmacal Research, vol. 38, no. 6, pp. 1044-1053, 2015.

[110] A. Papadimitriou, E. B. M. I. Peixoto, K. C. Silva, J. M. Lopes de Faria, and J. B. Lopes de Faria, "Increase in AMPK brought about by cocoa is renoprotective in experimental diabetes mellitus by reducing NOX4/TGF $\beta-1$ signaling," The Journal of Nutritional Biochemistry, vol. 25, no. 7, pp. 773-784, 2014.

[111] M. S. A. Gurukar, S. Mahadevamma, and N. D. Chilkunda, "Renoprotective Effect of Coccinia indica Fruits and Leaves in Experimentally Induced Diabetic Rats," Journal of Medicinal Food, vol. 16, no. 9, pp. 839-846, 2013.

[112] X.-M. Meng, P. M.-K. Tang, J. Li, and H. Y. Lan, "TGF$\hat{\mathrm{I}}^{2} /$ Smad signaling in renal fibrosis," Frontiers in Physiology, vol. 6, 2015.

[113] Q. Gao, W. S. Qin, Z. H. Jia et al., "Rhein Improves Renal Lesion and Ameliorates Dyslipidemia in $\mathrm{db} / \mathrm{db}$ Mice with
Diabetic Nephropathy," Planta Medica, vol. 76, no. 1, pp. 27-33, 2010.

[114] S.-H. Yu, N. K. Dubey, W. S. Li et al., "Cordyceps militaris treatment preserves renal function in type 2 diabetic nephropathy mice," PLoS One, vol. 11, no. 11, article e0166342, 2016.

[115] E. Seo, H. Kang, Y. Oh, and H.-S. Jun, "Psoralea corylifolia L. seed extract attenuates diabetic nephropathy by inhibiting renal fibrosis and apoptosis in streptozotocin-induced diabetic mice," Nutrients, vol. 9, no. 8, p. 828, 2017.

[116] Z.-R. Wang, H.-Y. Zhang, M.-F. Guo, Z.-X. Gao, and J.-L. Li, "Renal protection of Tangke decoction on rats with diabetes and its effect on the expression of TGF-beta1/Smad4," Zhongguo Zhong xi yi jie he za zhi Zhongguo Zhongxiyi jiehe zazhi, vol. 34, no. 7, pp. 826-832, 2014.

[117] W.-J. Ni, H.-H. Ding, H. Zhou, Y.-Y. Qiu, and L.-Q. Tang, "Renoprotective effects of berberine through regulation of the MMPs/TIMPs system in streptozocin-induced diabetic nephropathy in rats," European Journal of Pharmacology, vol. 764, pp. 448-456, 2015.

[118] Y.-Y. Qiu, L.-Q. Tang, and W. Wei, "Berberine exerts renoprotective effects by regulating the AGEs-RAGE signaling pathway in mesangial cells during diabetic nephropathy," Molecular and Cellular Endocrinology, vol. 443, pp. 89-105, 2017.

[119] Z. J. Chen, F. Ma, X. M. Sun, X. S. Zhao, and R. Luo, "Renoprotective Effect of a Chinese Herbal Formula, Qidan Dihuang Decoction, on Streptozotocin-Induced Diabetes in Rat," Evidence-Based Complementary and Alternative Medicine, vol. 2018, Article ID 7321086, 12 pages, 2018.

[120] Y. Wu, K. Ren, C. Liang et al., "Renoprotective Effect of Total Glucosides of Paeony (TGP) and Its Mechanism in Experimental Diabetes," Journal of Pharmacological Sciences, vol. 109, no. 1, pp. 78-87, 2009.

[121] F. L. Wang, L. Q. Tang, F. Yang, L. N. Zhu, M. Cai, and W. Wei, "Renoprotective effects of berberine and its possible molecular mechanisms in combination of high-fat diet and low-dose streptozotocin-induced diabetic rats," Molecular Biology Reports, vol. 40, no. 3, pp. 2405-2418, 2013.

[122] W.-J. Ni, L.-Q. Tang, H. Zhou, H.-H. Ding, and Y.-Y. Qiu, "Renoprotective effect of berberineviaregulating the PGE2EP1-G $\alpha$ q-Ca2+signalling pathway in glomerular mesangial cells of diabetic rats," Journal of Cellular and Molecular Medicine, vol. 20, no. 8, pp. 1491-1502, 2016.

[123] H. Zhao, X. Li, T. Zhao et al., "Tangshen formula attenuates diabetic renal injuries by upregulating autophagy via inhibition of PLZF expression," PloS One, vol. 12, no. 2, article e0171475, 2017.

[124] P. Li, Y. Chen, J. Liu et al., "Efficacy and Safety of Tangshen Formula on Patients with Type 2 Diabetic Kidney Disease: A Multicenter Double-Blinded Randomized PlaceboControlled Trial," PLoS One, vol. 10, no. 5, article e0126027, 2015.

[125] X. Yang, B. Zhang, X. Lu et al., "Effects of Tangshen Formula on urinary and plasma liver-type fatty acid binding protein levels in patients with type 2 diabetic kidney disease: posthoc findings from a multi-center, randomized, double-blind, placebo-controlled trial investigating the efficacy and safety of Tangshen Formula in patients with type 2 diabetic kidney disease," BMC Complementary and Alternative Medicine, vol. 16, no. 1, 2016. 
[126] H. Sasaki, A. Kamijo-Ikemori, T. Sugaya et al., "Urinary fatty acids and liver-type fatty acid binding protein in diabetic nephropathy," Nephron Clinical Practice, vol. 112, no. 3, pp. c148-c156, 2009.

[127] L. Peng, J. Yang, C. Ning et al., "Rhein Inhibits IntegrinLinked Kinase Expression and Regulates Matrix Metalloproteinase-9/Tissue Inhibitor of Metalloproteinase-1 Ratio in High Glucose-Induced Epithelial-Mesenchymal Transition of Renal Tubular Cell," Biological and Pharmaceutical Bulletin, vol. 35, no. 10, pp. 1676-1685, 2012.

[128] X. Tian, Z. Liu, B. Niu et al., "E-Cadherin/ $\beta$-Catenin Complex and the Epithelial Barrier," Journal of Biomedicine and Biotechnology, vol. 2011, Article ID 567305, 6 pages, 2011.

[129] Z. Lv, M. Hu, J. Zhen, J. Lin, Q. Wang, and R. Wang, "Rac1/PAK1 signaling promotes epithelial-mesenchymal transition of podocytes in vitro via triggering $\beta$-catenin transcriptional activity under high glucose conditions," The International Journal of Biochemistry \& Cell Biology, vol. 45, no. 2, pp. 255-264, 2013.

[130] L.-n. Sun, Z.-x. Chen, X.-c. Liu, H.-y. Liu, G.-j. Guan, and G. Liu, "Curcumin ameliorates epithelial-to-mesenchymal transition of podocytes in vivo and in vitro via regulating caveolin-1," Biomedicine \& Pharmacotherapy, vol. 68, no. 8, pp. 1079-1088, 2014.

[131] X. Wang, Y. Gao, N. Tian et al., “Astragaloside IV represses high glucose-induced mesangial cells activation by enhancing autophagy via SIRT1 deacetylation of NF- $\kappa$ B P65 subunit," Drug Design, Development and Therapy, vol. Volume 12, pp. 2971-2980, 2018.

[132] Y. Wei, J. Gao, L. Qin et al., "Curcumin suppresses AGEs induced apoptosis in tubular epithelial cells via protective autophagy," Experimental and Therapeutic Medicine, vol. 14, no. 6, pp. 6052-6058, 2017.

[133] X. H. Xu, D. F. Ding, H. J. Yong et al., "Resveratrol transcriptionally regulates miRNA-18a-5p expression ameliorating diabetic nephropathy via increasing autophagy," European Review for Medical and Pharmacological Sciences, vol. 21, no. 21, pp. 4952-4965, 2017.

[134] K. Yamahara, M. Yasuda, S. Kume, D. Koya, H. Maegawa, and T. Uzu, "The role of autophagy in the pathogenesis of diabetic nephropathy," Journal of Diabetes Research, vol. 2013, Article ID 193757, 9 pages, 2013.

[135] W.-J. Liu, W.-F. Huang, L. Ye et al., "The activity and role of autophagy in the pathogenesis of diabetic nephropathy," European Review Medical Pharmacological Sciences, vol. 22, pp. 3182-3189, 2018.

[136] Y. Jin, S. Liu, Q. Ma, D. Xiao, and L. Chen, "Berberine enhances the AMPK activation and autophagy and mitigates high glucose-induced apoptosis of mouse podocytes," European Journal of Pharmacology, vol. 794, pp. 106-114, 2017.

[137] F. Wu, S. Li, N. Zhang et al., "Hispidulin alleviates highglucose-induced podocyte injury by regulating protective autophagy," Biomedicine \& Pharmacotherapy, vol. 104, pp. 307-314, 2018.

[138] H. Zhan, J. Jin, S. Liang, L. Zhao, J. Gong, and Q. He, “Tripterygium glycoside protects diabetic kidney disease mouse serum-induced podocyte injury by upregulating autophagy and downregulating $\beta$-arrestin-1," Histology and Histopathology, vol. 34, no. 8, pp. 943-952, 2019.

[139] X. Wang, L. Gao, H. Lin et al., "Mangiferin prevents diabetic nephropathy progression and protects podocyte function via autophagy in diabetic rat glomeruli," European Journal of Pharmacology, vol. 824, pp. 170-178, 2018.

[140] F. Chen, Z. Sun, X. Zhu, and Y. Ma, “Astilbin inhibits high glucose-induced autophagy and apoptosis through the PI3K/Akt pathway in human proximal tubular epithelial cells," Biomedicine \& Pharmacotherapy, vol. 106, pp. 11751181, 2018.

[141] X. Li, S. S. Wang, Z. Han et al., "Triptolide restores autophagy to alleviate Diabetic Renal Fibrosis through the miR-1413p/PTEN/Akt/mTOR pathway," Molecular Therapy-Nucleic Acids, vol. 9, pp. 48-56, 2017.

[142] X.-X. Zhang, C.-H. Jiang, Y. Liu et al., “_Cyclocarya paliurus_ triterpenic acids fraction attenuates kidney injury via AMPKmTOR-regulated autophagy pathway in diabetic rats," Phytomedicine, vol. 64, article 153060, 2019.

[143] H. Kim, T. Dusabimana, S. R. Kim et al., "Supplementation of Abelmoschus manihot ameliorates diabetic nephropathy and hepatic steatosis by activating autophagy in mice," Nutrients, vol. 10, no. 11, p. 1703, 2018.

[144] L. Ma, R. Fu, Z. Duan et al., "Sirt1 is essential for resveratrol enhancement of hypoxia-induced autophagy in the type 2 diabetic nephropathy rat," Pathology - Research and Practice, vol. 212, no. 4, pp. 310-318, 2016.

[145] S. Chowdhury, S. Ghosh, A. K. Das, and P. C. Sil, "Ferulic acid protects hyperglycemia-induced kidney damage by regulating oxidative insult, inflammation and autophagy," Frontiers in Pharmacology, vol. 10, 2019.

[146] S. Sato, S. Kataoka, A. Kimura, and Y. Mukai, "Azuki bean (Vigna angularis) extract reduces oxidative stress and stimulates autophagy in the kidneys of streptozotocin-induced early diabetic rats," Canadian Journal of Physiology and Pharmacology, vol. 94, no. 12, pp. 1298-1303, 2016.

[147] S. Kume, T. Uzu, K. Isshiki, and D. Koya, "Peroxisome proliferator-activated receptors in diabetic nephropathy," PPAR Research, vol. 2008, Article ID 879523, 11 pages, 2008.

[148] N. Nakagawa, K. Yuhki, J. Kawabe et al., “The intrinsic prostaglandin $\mathrm{E}_{2}-\mathrm{EP}_{4}$ system of the renal tubular epithelium limits the development of tubulointerstitial fibrosis in mice," Kidney International, vol. 82, no. 2, pp. 158-171, 2012.

[149] R. Hu, C. L.-L. Saw, R. Yu, and A. N. T. Kong, "Regulation of NF-E2-related factor 2 signaling for cancer chemoprevention: antioxidant coupled with antiinflammatory," Antioxidants \& Redox Signaling, vol. 13, no. 11, pp. 1679-1698, 2010.

[150] J. R. Sowers, "Endocrine functions of adipose tissue: focus on adiponectin," Clinical Cornerstone, vol. 9, no. 1, pp. 32-40, 2008.

[151] R. Seger and E. G. Krebs, "The MAPK signaling cascade," FASEB Journal, vol. 9, no. 9, pp. 726-735, 1995.

[152] Y. Wang, S. Huang, V. P. Sah et al., "Cardiac muscle cell hypertrophy and apoptosis induced by distinct members of the p38 mitogen-activated protein Kinase family," Journal of Biological Chemistry, vol. 273, no. 4, pp. 2161-2168, 1998.

[153] X. Li, W. Liu, Q. Wang et al., "Emodin suppresses cell proliferation and fibronectin expression via p38MAPK pathway in rat mesangial cells cultured under high glucose," Molecular and Cellular Endocrinology, vol. 307, no. 1-2, pp. 157-162, 2009.

[154] X. Yang, X. Xiao, H. Wang et al., "Renoprotective Effect of Danhong Injection on Streptozotocin-Induced Diabetic Rats through a Peroxisome Proliferator-Activated Receptor $\gamma$ Mediated Pathway," Evidence-Based Complementary and 
Alternative Medicine, vol. 2018, Article ID 3450141, 10 pages, 2018.

[155] X. Yang, Y. Li, C. Feng, L. Guo, and H. Jin, "In Vivo Study of the Renoprotective Effects of EGCG in Diabetic db/db Mice," Zhonghua Yi Xue Za Zhi, vol. 96, no. 17, pp. 1330-1335, 2016.

[156] Z.-M. Mao, S.-M. Shen, Y.-G. Wan et al., "Huangkui capsule attenuates renal fibrosis in diabetic nephropathy rats through regulating oxidative stress and p38MAPK/Akt pathways, compared to $\alpha$-lipoic acid," Journal of Ethnopharmacology, vol. 173, pp. 256-265, 2015.

[157] N. Kandasamy and N. Ashokkumar, "Renoprotective effect of myricetin restrains dyslipidemia and renal mesangial cell proliferation by the suppression of sterol regulatory element binding proteins in an experimental model of diabetic nephropathy," European Journal of Pharmacology, vol. 743, pp. 53-62, 2014.

[158] Y. Yang, W. Ni, M. Cai, L. Tang, and W. Wei, "The renoprotective effects of berberineviathe EP4-Gas-cAMP signaling pathway in different stages of diabetes in rats," Journal of Receptors and Signal Transduction, vol. 34, no. 6, pp. 445455, 2014.

[159] L. Zhang, J. L. Shergis, L. Yang et al., “Astragalus membranaceus (Huang Qi) as adjunctive therapy for diabetic kidney disease: An updated systematic review and meta-analysis," Journal of Ethnopharmacology, vol. 239, article 111921, 2019.

[160] M. Wang, Z. Wang, J. Zhou et al., "Effects of traditional Chinese herbal medicine in patients with diabetic kidney disease: study protocol for a randomized controlled trial," Trials, vol. 19, no. 1, p. 389, 2018.

[161] X. Zhao, Y. Zhang, X. H. He et al., "Chinese herbal medicine Shenzhuo Formula treatment in patients with macroalbuminuria secondary to diabetic kidney disease: study protocol for a randomized controlled trial," Trials, vol. 19, no. 1, p. 200, 2018.

[162] D. Jin, W. J. Huang, X. Meng et al., "Chinese herbal medicine Tangshen Formula treatment for type 2 diabetic kidney disease in the early stage: study protocol for a randomized controlled trial," Trials, vol. 20, no. 1, p. 756, 2019.

[163] H. Wang, W. Mu, J. Zhai et al., "The key role of Shenyan Kangfu tablets, a Chinese patent medicine for diabetic nephropathy: study protocol for a randomized, doubleblind and placebo-controlled clinical trial," Trials, vol. 14, no. 1, p. 165, 2013.

[164] M. Yan, Y. Wen, L. Yang et al., "Chinese herbal medicine Tangshen Formula treatment of patients with type 2 diabetic kidney disease with macroalbuminuria: Study protocol for a randomized controlled trial," Trials, vol. 17, no. 1, p. 259, 2016.

[165] T.-z. Wang, Y. Chen, Y.-m. He et al., "Effects of Chinese herbal medicine Yiqi Huaju Qingli Formula in metabolic syndrome patients with microalbuminuria: a randomized placebo-controlled trial," Journal of Integrative Medicine, vol. 11, no. 3, pp. 175-183, 2013.

[166] A. M. Aleali, R. Amani, H. Shahbazian, F. Namjooyan, S. M. Latifi, and B. Cheraghian, "The effect of hydroalcoholic Saffron (Crocus sativus L.) extract on fasting plasma glucose, $\mathrm{HbA1c}$, lipid profile, liver, and renal function tests in patients with type 2 diabetes mellitus: a randomized double-blind clinical trial," Phytotherapy Research, vol. 33, no. 6, pp. 1648-1657, 2019.
[167] L. Zhang, L. Yang, J. Shergis et al., "Chinese herbal medicine for diabetic kidney disease: a systematic review and metaanalysis of randomised placebo-controlled trials," BMJ Open, vol. 9, no. 4, article e025653, 2019.

[168] S. S. Huang, D. F. Ding, S. Chen et al., "Resveratrol protects podocytes against apoptosis via stimulation of autophagy in a mouse model of diabetic nephropathy," Scientific Reports, vol. 7, no. 1, 2017.

[169] K. S. Kang, N. Yamabe, H. Y. Kim, J. H. Park, and T. Yokozawa, "Therapeutic potential of 20(S)-ginsenoside $\mathrm{Rg}_{3}$ against streptozotocin-induced diabetic renal damage in rats," European Journal of Pharmacology, vol. 591, no. 1-3, pp. 266-272, 2008.

[170] X. Zhan, C. Yan, Y. Chen et al., "Celastrol antagonizes high glucose-evoked podocyte injury, inflammation and insulin resistance by restoring the HO-1-mediated autophagy pathway," Molecular Immunology, vol. 104, pp. 61-68, 2018.

[171] T. Shiju, N. Rajesh, and P. Viswanathan, "Renoprotective effect of aged garlic extract in streptozotocin-induced diabetic rats," Indian Journal of Pharmacology, vol. 45, no. 1, pp. 18-23, 2013.

[172] P. K. Singh, D. Baxi, A. Doshi, and R. A V, “Antihyperglycaemic and renoprotective effect of Boerhaavia diffusa L. in experimental diabetic rats," Journal of Complementary and Integrative Medicine, vol. 8, no. 1, 2011.

[173] T. Senthilkumar, N. Sangeetha, and N. Ashokkumar, "Antihyperglycemic, antihyperlipidemic, and renoprotective effects of Chlorella pyrenoidosa in diabetic rats exposed to cadmium," Toxicology Mechanisms and Methods, vol. 22, no. 8, pp. 617-624, 2012.

[174] M. Mirmohammadlu, S. H. Hosseini, M. Kamalinejad, M. Esmaeili Gavgani, M. Noubarani, and M. R. Eskandari, "Hypolipidemic, hepatoprotective and renoprotective effects of Cydonia oblonga Mill. fruit in streptozotocin-induced diabetic rats," Iranian Journal of Pharmaceutical Research, vol. 14, no. 4, pp. 1207-1214, 2015.

[175] S. Hamzeh, F. Farokhi, R. Heydari, and R. Manaffar, "Renoprotective effect of hydroalcoholic extract of Rheum ribes root in diabetic female rats," Avicenna Journal of Phytomedicine, vol. 4, no. 6, pp. 392-401, 2014.

[176] A. Ontawong, N. Saowakon, P. Vivithanaporn et al., "Antioxidant and renoprotective effects of Spirogyra neglecta (Hassall) Kützing extract in experimental type 2 diabetic rats," BioMed Research International, vol. 2013, Article ID 820786, 15 pages, 2013. 